\title{
A Flexible Parameterization for Shortwave Optical Properties of Ice Crystals*
}

\author{
BASTIAAN VAN DIEDENHOVEN \\ Center for Climate System Research, Columbia University, and NASA Goddard Institute for Space Research, \\ New York, New York \\ Andrew S. ACKERman, Brian CAIRns, AND ANn M. FridLind \\ NASA Goddard Institute for Space Research, New York, New York
}

(Manuscript received 1 July 2013, in final form 21 November 2013)

\begin{abstract}
A parameterization is presented that provides extinction cross section $\sigma_{e}$, single-scattering albedo $\omega$, and asymmetry parameter $g$ of ice crystals for any combination of volume, projected area, aspect ratio, and crystal distortion at any wavelength in the shortwave. Similar to previous parameterizations, the scheme makes use of geometric optics approximations and the observation that optical properties of complex, aggregated ice crystals can be well approximated by those of single hexagonal crystals with varying size, aspect ratio, and distortion levels. In the standard geometric optics implementation used here, $\sigma_{e}$ is always twice the particle projected area. It is shown that $\omega$ is largely determined by the newly defined absorption size parameter and the particle aspect ratio. These dependences are parameterized using a combination of exponential, lognormal, and polynomial functions. The variation of $g$ with aspect ratio and crystal distortion is parameterized for one reference wavelength using a combination of several polynomials. The dependences of $g$ on refractive index and $\omega$ are investigated and factors are determined to scale the parameterized $g$ to provide values appropriate for other wavelengths. The parameterization scheme consists of only 88 coefficients. The scheme is tested for a large variety of hexagonal crystals in several wavelength bands from 0.2 to $4 \mu \mathrm{m}$, revealing absolute differences with reference calculations of $\omega$ and $g$ that are both generally below 0.015 . Over a large variety of cloud conditions, the resulting root-mean-squared differences with reference calculations of cloud reflectance, transmittance, and absorptance are $1.4 \%, 1.1 \%$, and $3.4 \%$, respectively. Some practical applications of the parameterization in atmospheric models are highlighted.
\end{abstract}

\section{Introduction}

A substantial uncertainty in properly representing ice clouds and consequently their climate feedbacks is characterizing their radiative properties (Stephens et al. 1990; Stackhouse and Stephens 1991; Schlimme et al. 2005; Fu 2007). The fundamental radiative properties of atmospheric ice crystals for atmospheric models are the ice extinction cross section, single-scattering albedo, and the first moment of the scattering phase function, commonly referred to as the asymmetry parameter

\footnotetext{
* Supplemental information related to this paper is available at the Journals Online website: http://dx.doi.org/10.1175/JAS-D-130205.s1.

Corresponding author address: Bastiaan van Diedenhoven, 2880 Broadway, New York, NY 10025.

E-mail: bastiaan.vandiedenhoven@nasa.gov
}

DOI: 10.1175/JAS-D-13-0205.1

(C) 2014 American Meteorological Society
(Coakley and Chylek 1975; Fu 1996; Yang et al. 2000; Fu 2007). An increasing number of parameterizations for these optical properties of ice clouds are available. Such parameterizations generally relate the optical properties in selected wavelength bands in terms of predicted or imposed bulk characteristics of the ice, such as effective size, shape, and ice water content (e.g., Fu and Liou 1993; Fu 1996; Wyser and Yang 1998; Kristjánsson et al. 1999; Yang et al. 2000; McFarquhar et al. 2002; Key et al. 2002; Edwards et al. 2007; Xie et al. 2012), sometimes in combination with atmospheric conditions, such as temperature and ice supersaturation (Kristjánsson et al. 2000; Baran 2012). Although ice crystals are found with a virtually countless variation of shapes (Baran 2009), the extinction cross section and single-scattering albedo of randomly oriented individual ice crystals mainly depend on their projected areas and volumes, while the asymmetry parameter is shown to be determined mainly by the aspect ratios (defined throughout as the ratio of 
hexagonal prism height to width) of the ice crystal hexagonal components and their microscale surface roughness or crystal distortion (Iaquinta et al. 1995; Macke et al. 1996; Fu 2007; Yang and Fu 2009; Baran 2009; van Diedenhoven et al. 2012a).

Parallel to advances in understanding ice optical properties are efforts to improve ice microphysics modeling schemes. Some of these schemes are becoming increasingly complex and calculate or parameterize ice fall speeds and capacitances (an electrostatic analog used to compute ice deposition and sublimation rates) in terms of ice mass, projected area, and aspect ratio (Böhm 1989, 1992; Heymsfield and Iaquinta 2000; Wood et al. 2001; Westbrook 2008; Westbrook et al. 2008; Sulia and Harrington 2011; van Diedenhoven et al. 2012b). Since mass $m$ can be related to bulk ice volume $V$ via $V=m / \rho_{i}$, where $\rho_{i}$ is the density of bulk ice, the ice characteristics determining fall speeds and capacitances are largely the same as those determining ice optical properties. Only crystal distortion does not affect fall speeds and capacitances substantially while being important for ice crystal optical properties. Selfconsistency within a model dictates that the same ice volume, area, and aspect ratio used in an ice microphysics scheme should also be used in a model's radiative transfer scheme (Mitchell et al. 2008; Baran 2012). However, there is at present a lack of optical property parameterizations flexible enough to accommodate all possible combinations of ice volume, area, aspect ratio, and crystal distortion. Furthermore, optical properties parameterizations are usually given for a predetermined set of wavelength bands, which are not necessarily the same as those used in a model's radiative transfer calculations. Finally, most current optical property schemes require many and large tables to be read by the radiative transfer algorithm.

In this paper, we present a simple yet flexible parameterization that provides the extinction cross section, single-scattering albedo, and asymmetry parameter of ice crystals for any combination of volume, projected area, aspect ratio (of crystal components), and crystal distortion and at any wavelength in the shortwave. Similar to previous parameterizations (e.g., Fu 1996, 2007), our scheme makes use of geometric optics approximations and the observation that optical properties of complex, aggregated ice crystals can be approximated by those of single hexagonal crystals with varying size, aspect ratio, and crystal distortion.

After describing the reference calculations, the theory, and the parameterization in section 2, we assess its accuracy in section 3. We provide an example of an application in section 4 and present conclusions in section 5 .

\section{Theory and parameterizations}

Here, we first discuss the dependencies of extinction cross section, absorption cross section, and asymmetry parameter on ice crystal volume, projected area, aspect ratio, and crystal distortion before parameterizing those dependencies.

\section{a. Reference calculations}

The reference calculations are based on single hexagonal plates and columns. The optical properties for hexagonal plates and columns with random orientation are calculated using the geometric optics (GO) code developed by Macke et al. (1996). In this standard geometric optics implementation, the extinction cross section is twice the projected area of crystals in random orientation. This Monte Carlo ray-tracing code takes crystal distortion of ice crystals into account in a statistical manner by perturbing the normal of the crystal surface from its nominal orientation by an angle that, for each interaction with a ray, is varied randomly with uniform distribution between $0^{\circ}$ and $\delta \times 90^{\circ}$, where $\delta$ is referred to as the distortion parameter. Thus, $\delta$ does not represent a single realization of a distorted crystal but rather the stochastic large-scale distortion of a collection of ice crystals (Macke et al. 1996). For a large collection of ice crystals, microscale surface roughness and largescale particle distortion both lead to a similar randomization of the angles between crystal facets, which in turn leads to the suppression of features in the scattering phase matrix (Yang et al. 2008; Neshyba et al. 2013). Increasing the number of impurities within ice crystals also has a similar effect (Hess et al. 1998). Thus, the distortion parameter used here can be considered as a proxy for the randomization of the angles between crystal facets caused by any of these effects.

Calculations are made for bands with the average wavelengths and complex refractive indices listed in Table 1. Various sets of calculations including different dimensions of the hexagonal particles are used to construct and evaluate the parameterization, as detailed in the next subsections. The relations between volume $V$, orientation-averaged projected area $A_{p}$, maximum halfwidth of the hexagonal planes $a_{\text {hex }}$, and aspect ratio $\alpha$ of hexagonal plates and columns are given in appendix A.

The reference calculations and thus the parameterization presented here are based on conventional geometric optics calculations. It is generally accepted that GO approximations are applicable to scattering size parameters above about 100 (e.g., Macke et al. 1995; Yang et al. 2000, 2004, 2013). (Here we define the particle scattering size parameter $\chi_{\text {scat }}$ as $2 \pi r_{\text {sph }} / \lambda$, where $r_{\text {sph }}$ is the radius of a sphere with the equivalent projected 
TABLE 1 . Average wavelengths $\lambda_{a}$ and real $\left(m_{r}\right)$ and imaginary $\left(m_{i}\right)$ parts of the refractive index used in the reference calculations. Values of $m_{r}, m_{i}$ (Warren 1984; Warren and Brandt 2008), and $\lambda_{a}$ are weighted by solar irradiance at the top of the atmosphere within each wavelength band. Band edges are given in Table 1 of Kato et al. (1999), with bands 9-15 there corresponding to band 9 here.

\begin{tabular}{ccc}
\hline \hline$\lambda_{a}(\mu \mathrm{m})$ & $m_{r}$ & $m_{i}$ \\
\hline 0.256 & 1.3480 & $8.082 \times 10^{-9}$ \\
0.280 & 1.3407 & $6.751 \times 10^{-9}$ \\
0.296 & 1.3353 & $5.756 \times 10^{-9}$ \\
0.319 & 1.3307 & $4.878 \times 10^{-9}$ \\
0.335 & 1.3275 & $4.269 \times 10^{-9}$ \\
0.365 & 1.3231 & $3.420 \times 10^{-9}$ \\
0.420 & 1.3177 & $2.261 \times 10^{-9}$ \\
0.482 & 1.3140 & $1.742 \times 10^{-9}$ \\
0.598 & 1.3098 & $7.511 \times 10^{-9}$ \\
0.690 & 1.3071 & $2.445 \times 10^{-8}$ \\
0.719 & 1.3056 & $7.549 \times 10^{-8}$ \\
0.762 & 1.3065 & $3.834 \times 10^{-8}$ \\
0.813 & 1.3047 & $1.376 \times 10^{-7}$ \\
0.862 & 1.3038 & $2.330 \times 10^{-7}$ \\
0.926 & 1.3028 & $5.267 \times 10^{-7}$ \\
1.005 & 1.3014 & $1.724 \times 10^{-6}$ \\
1.111 & 1.2997 & $2.228 \times 10^{-6}$ \\
1.333 & 1.2955 & $7.335 \times 10^{-5}$ \\
1.562 & 1.2906 & $4.841 \times 10^{-4}$ \\
1.770 & 1.2837 & $2.627 \times 10^{-4}$ \\
2.051 & 1.2717 & $1.212 \times 10^{-3}$ \\
2.210 & 1.2629 & $3.064 \times 10^{-4}$ \\
2.584 & 1.1815 & $2.773 \times 10^{-2}$ \\
3.284 & 1.4310 & $2.719 \times 10^{-1}$ \\
3.809 & 1.3874 & $7.558 \times 10^{-3}$ \\
4.292 & 1.3473 & $1.639 \times 10^{-2}$ \\
\hline & &
\end{tabular}

area; i.e., $r_{\mathrm{sph}}=\sqrt{A_{p} / \pi}$.) For smaller sizes, the errors on ice crystal optical properties and ice cloud radiation calculations caused by GO approximations are not yet adequately quantified. Such a quantification would require intensive calculations using techniques that are able to accurately calculate optical properties of a variety of ice crystal shapes with small size parameters (e.g., Yang et al. 2013). Such an investigation is beyond the scope of the current paper and left for future work.

\section{b. Extinction cross section and single-scattering albedo}

The single-scattering albedo of a single ice particle is defined to be

$$
\omega=1-\frac{\sigma_{a}}{\sigma_{e}}
$$

where the extinction cross section of a particle is given by

$$
\sigma_{e}=Q_{e} A_{p},
$$

where $Q_{e}$ is the extinction efficiency, which is equal to 2 in the geometric optics limit (Macke et al. 1996; Yang and Liou 1996). According to anomalous diffraction theory (ADT; van de Hulst 1957; Bryant and Latimer 1969; Mitchell and Arnott 1994), the absorption cross section can be approximated by

$$
\sigma_{a} \approx\left[1-\exp \left(\frac{-4 \pi m_{i} V}{\lambda A_{p}}\right)\right] A_{p},
$$

where $m_{i}$ is the imaginary part of the refractive index (Table 1 ) at wavelength $\lambda$, and $V$ is the volume of the ice crystal, which is related to mass $m$ via $V=m / \rho i$. From Eqs. (1)-(3), it is apparent that the single-scattering albedo at a given wavelength is mainly determined by the ice crystal effective distance or photon path $d_{e}$ as given by Bryant and Latimer (1969):

$$
d_{e}=\frac{V}{A_{p}} .
$$

The relation between the single-scattering albedo of a compact hexagonal particle with aspect ratio $\alpha$ of unity and the effective distance for the wavelength bands with substantial absorption is shown in Fig. 1a. For this, calculations were made for smooth crystals with 100 different ratios of volume to projected area varying between 1 and $135 \mu \mathrm{m}$. The effect of particle distortion on singlescattering albedo is considered negligible (Yi et al. 2013).

The ice crystal effective distance is related to the commonly used, but variably defined (McFarquhar and Heymsfield 1998) bulk ice effective diameter $D_{e}$, in which "bulk" denotes an average over a particle size distribution. Here we define $D_{e}$ through the ratio of the size-distribution integrated volume and project area:

$$
D_{e}=\frac{3}{2} \frac{\int_{0}^{\infty} V(D) N(D) d D}{\int_{0}^{\infty} A_{p}(D) N(D) d D},
$$

where $N$ is the number density of the ice crystals of maximum dimension $D$ (Foot 1988; Francis et al. 1994; Baum et al. 2005a). The smooth relation of the singlescattering albedo to the ratio of volume to area has often been used to parameterize the single-scattering albedo as a function of bulk ice effective diameter (e.g., Wyser and Yang 1998; Yang et al. 2000; McFarquhar et al. 2002; Key et al. 2002; Edwards et al. 2007; Xie et al. 2012).

However, Eq. (3) suggests that a more fundamental parameter governing the single-scattering albedo of individual particles is

$$
\chi_{\mathrm{abs}}=\frac{m_{i} V}{\lambda A_{p}}=\frac{m_{i}}{\lambda} d_{e},
$$




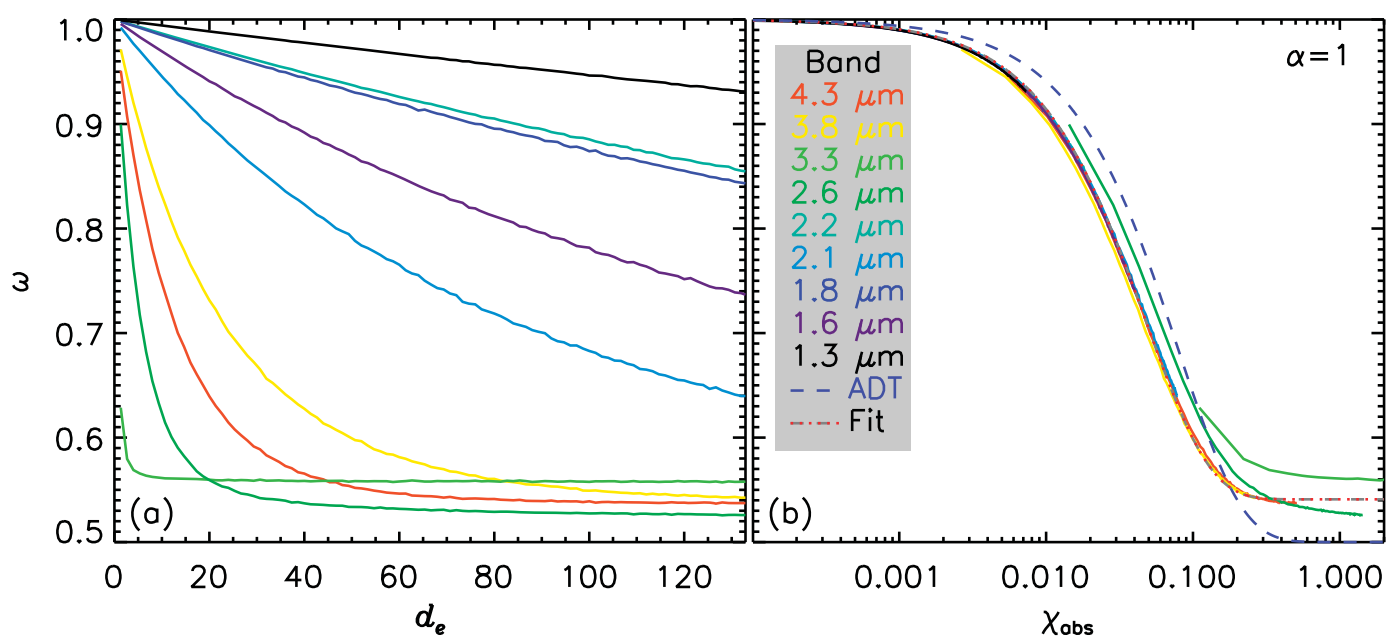

FIG. 1. Single-scattering albedo for compact hexagonal ice crystals as a function of (a) effective distance and (b) absorption size parameter. Colors indicate different wavelength bands. The gray-red line represents the exponential fit described in the text. The blue dashed line is the result using ADT.

which we will refer to as the absorption size parameter. Note that $\chi_{\text {abs }}$ is dimensionless. As seen in Fig. 1b, the single-scattering albedo in nearly all bands collapse to a single function of $\chi_{\text {abs. }}$. Only single-scattering albedos corresponding to the two bands with the strongest absorption (2.6 and $3.3 \mu \mathrm{m}$ with $m_{i}>0.02$ as seen in Table 1) substantially deviate from the single curve. For the remaining bands, single-scattering albedos at the same absorption size parameter but for different wavelengths agree within about 0.01 , which is discussed in greater detail later. The dependence of single-scattering albedo for compact particles $\left(\omega_{\alpha=1}\right)$ to absorption size parameter is found to be fit well by an exponential function:

$$
\omega_{\alpha=1}=1-a_{0}\left(1-e^{-a_{1} \chi_{\mathrm{abs}}}\right)
$$

where the form of the parameterization guarantees that $\omega_{\alpha=1}=1$ for $\chi_{\text {abs }}=0$. Using a least squares fit, we find $a_{0}=0.457593$ and $a_{1}=20.9738$. (The parameterization coefficients are computed in single precision with 4-byte floating points and given to six significant digits throughout.) The values corresponding to the 2.6- and 3.3- $\mu \mathrm{m}$ bands with strong absorption were excluded when performing this fit. The root-mean-square difference between the fit and the single-scattering albedos used for the fit is 0.002 . As evident from Fig. 1b, errors for strongly absorbing wavelengths are larger, as further discussed in section 3. As can be derived from Eqs. (1)-(3), in conventional ADT, the coefficients $a_{0}$ and $a_{1}$ of Eq. (7) are $1 / Q_{e}$ and $4 \pi$, respectively (Mitchell and Arnott 1994). However, Fig. 1b shows that ADT generally overestimates the single-scattering albedo of compact hexagonal crystals. As discussed by Mitchell et al. (1996) among others, this error is principally attributable to the neglect of internal reflections. Several modifications to ADT have been proposed (e.g., Mitchell 2002; Yang et al. 2004), which generally include several tuning factors; an evaluation of such schemes is beyond the scope of this study. Note that a quantity related to the absorption size parameter [Eq. (6)] was used previously by Takano and Liou (1989) to parameterize $\omega$ for weakly absorbing $(\omega<0.75)$ hexagonal crystals at wavelengths shorter than $2.5 \mu \mathrm{m}$.

The parameterization described by Eq. (7) assumes compact hexagonal ice crystals $(\alpha=1)$. When aspect ratios deviate from unity by about a factor of $2, \omega$ is generally greater than that of a compact hexagonal crystal, as seen in Fig. 2. This effect is stronger for plates (Fig. 2a) than for columns (Fig. 2b). Presumably this increase of $\omega$ with aspect ratio deviating from unity results from a decrease of the orientation-averaged pathlength through the particle, because internal reflections are maximized for compact particles. Similar to Fig. 1b, Fig. 2 shows that, for a given aspect ratio, $\omega$ collapses to a single function of $\chi_{\mathrm{abs}}$. Note however that slight differences in $\omega$ at the same $\chi$ abs but for different combinations of $d_{e}$ and wavelengths result in "spikes" in Fig. 2. Such spikes may result from wave resonance effects, leading to rays orbiting the particle through a series of internal reflections for specific combinations of wavelength, refractive index, and particle size, thereby increasing absorption (Guimarães and Nussenzveig 1992; Mitchell et al. 2006). Interestingly, the spikes diminish as aspect ratio increasingly deviates from unity, which is consistent with such ray-orbiting phenomena (Mitchell et al. 2006). However, some Monte Carlo noise is also apparent in Fig. 2, which increases with aspect ratio deviation from unity. Figure 2 also shows functional fits 


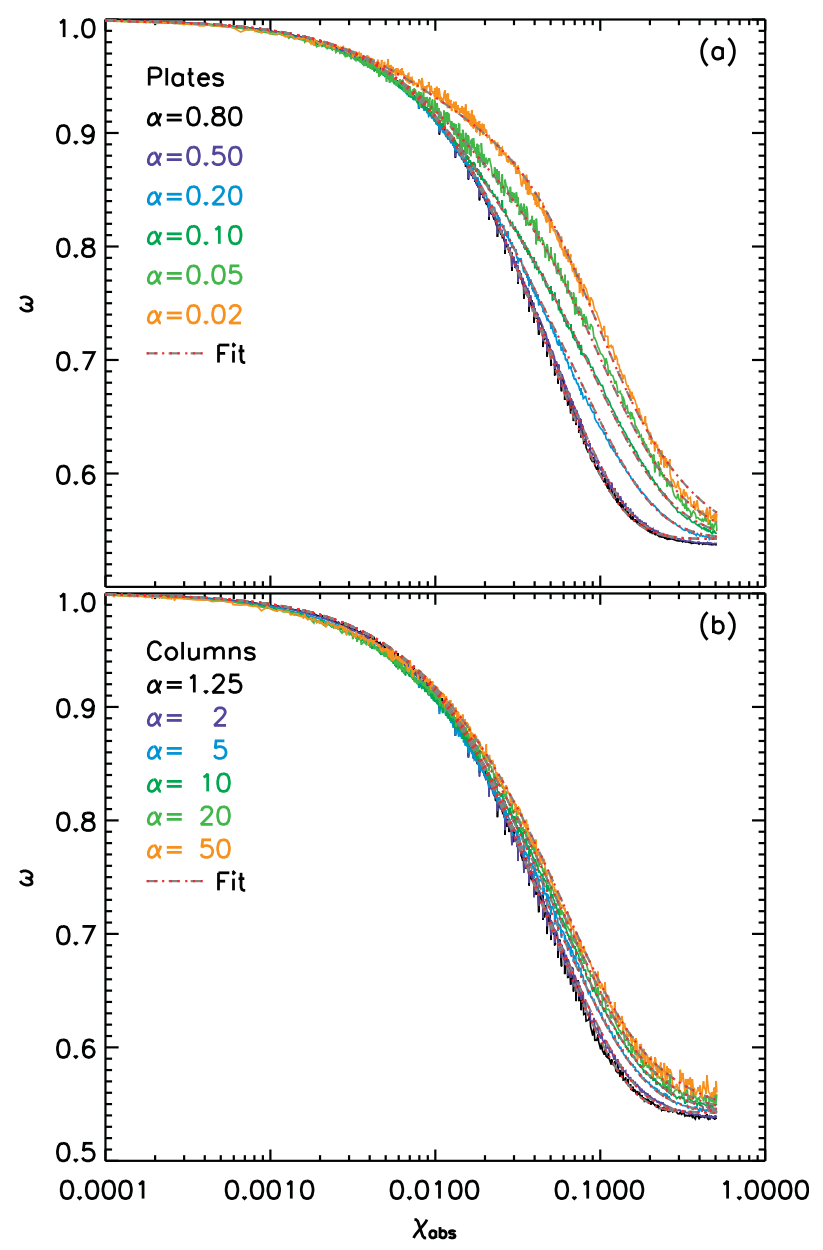

FIG. 2. As in Fig. 1b, but for (a) hexagonal plates and (b) columns with various aspect ratios, as indicated by the colors. All bands listed in Table 1 with weak to moderate absorption $\left(m_{i}<0.02\right)$ are included. Gray-red dashed lines indicate fits.

to the $\chi_{\text {abs }}$ versus- $\omega$ relations for plates with aspect ratios of $0.8,0.5,0.2,0.1,0.05$, and 0.02 and for columns with aspect ratios that are the inverse of those for the plates. First, the difference $\Delta \omega_{\alpha}$ between the calculated singlescattering albedo and the exponential fit assuming compact hexagonal crystals [Fig. $1 \mathrm{~b}$ and Eq. (7)] is determined for each of the aspect ratios, as shown in Fig. 3. We find that $\Delta \omega_{\alpha}$ can be approximated by lognormal functions that are also shown in Fig. 3; that is,

$$
\Delta \omega_{\alpha} \approx \frac{l_{0}}{\sqrt{2 \pi} l_{1} \chi_{\mathrm{abs}}} \exp \left[-\frac{\left(\ln \chi_{\mathrm{abs}}-l_{2}\right)^{2}}{2 l_{1}^{2}}\right] .
$$

The fit parameters $l_{i}$, where $i=0-2$, are determined for each of the aspect ratios using a least squares fitting method. In turn, $l_{i}$ are found to be smooth functions of the logarithm of $\alpha$ that, for either plates and columns, can be approximated by cubic functions; that is,

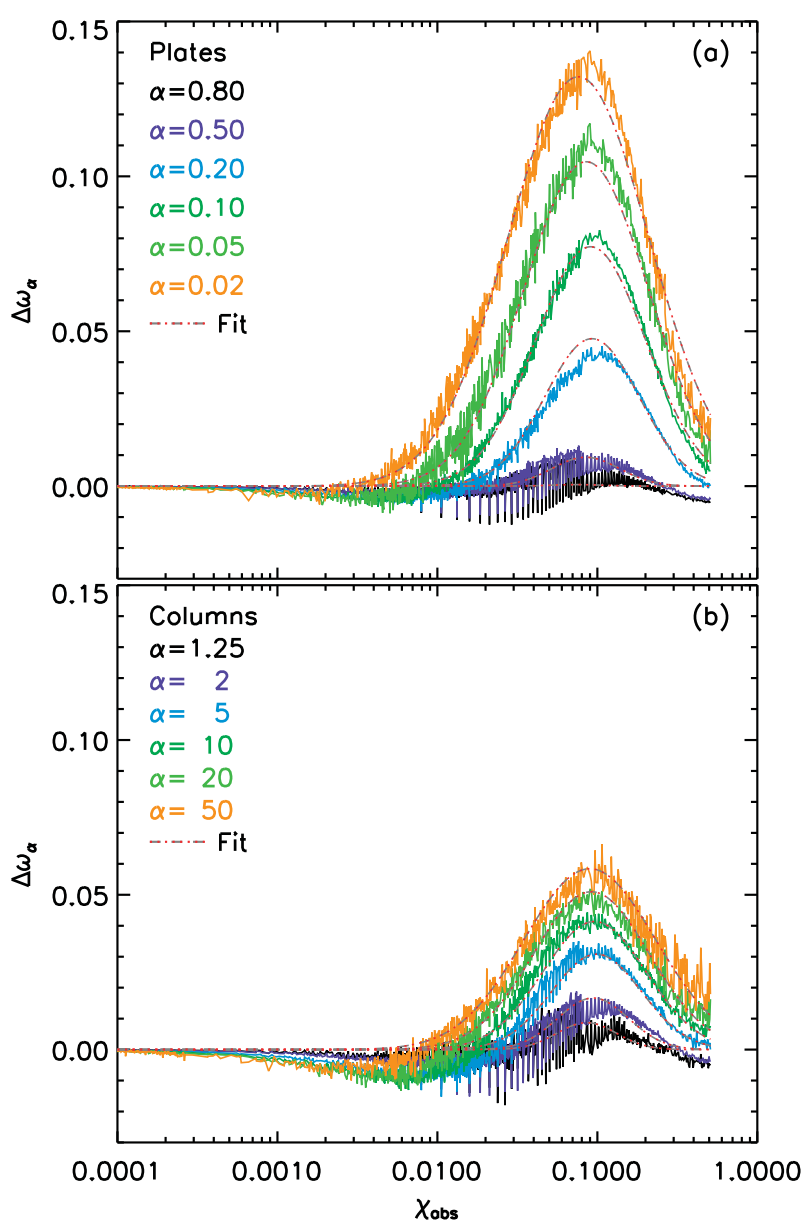

FIG. 3. Differences between the parameterized single-scattering albedo for particles with an aspect ratio of unity and the calculated single-scattering albedos for several (a) plates and (b) columns with various aspect ratios. Gray-red dashed lines indicate fitted lognormal functions as described in the text.

$$
l_{i}(\alpha)=\sum_{j=0}^{3} c_{i, j} \log ^{j} \alpha,
$$

for which the coefficients $c_{i, j}$ are listed in Table 2. (Throughout, "log" refers to base 10.) To better constrain this latter fit, we also include calculations for aspect ratios of 0.01 and 100, which are not shown in Figs. 2 and 3. Finally, the single-scattering albedo of a hexagonal ice crystal of any size and any aspect ratio can be estimated for any wavelength by

$$
\omega\left(\chi_{\mathrm{abs}}, \alpha\right)=\omega_{\alpha=1}+\Delta \omega_{\alpha},
$$

where $\omega_{\alpha=1}$ and $\Delta \omega_{\alpha}$ are calculated using Eqs. (7) and (8), respectively. Note that the spikes and noise seen in Fig. 2, as mentioned above, are even more apparent in Fig. 3. Given that such spikes are about $0.01 \omega$ units in 
TABLE 2. Coefficients $c_{i, j}$ used by Eq. (9) to compute coefficients $l_{i}$ in Eq. (8). See also Fig. 4, box 3.

\begin{tabular}{lllcc}
\hline \hline & $j$ & \multicolumn{1}{c}{$c_{0, j}$} & $c_{1, j}$ & $c_{2, j}$ \\
\hline Plates & 0 & 0.000527060 & 0.309748 & -2.58028 \\
& 1 & 0.00867596 & -0.650188 & -1.34949 \\
& 2 & 0.0382627 & -0.198214 & -0.674495 \\
& 3 & 0.0108558 & -0.0356019 & -0.141318 \\
Columns & 0 & 0.000378774 & 0.390452 & -2.36821 \\
& 1 & 0.00463283 & 0.420040 & 1.07603 \\
& 2 & 0.00593106 & -0.0848059 & -0.729980 \\
& 3 & -0.00117167 & 0.0186601 & 0.232446 \\
\hline
\end{tabular}

magnitude, the parameterization of $\omega$ (see Fig. 4 for a summary) is limited to an overall accuracy of about 0.01 at wavelengths where $m_{i}<0.02$. The resulting parameterized single-scattering albedo as a function of the absorption size parameter and aspect ratios for plates and columns is shown in Fig. 5.

As given by Eq. (2), the extinction cross section is always twice the projected area of crystals in random orientation in the standard geometric optics implementation used here (see Fig. 4, box 5). This approximation generally holds for scattering size parameters above about 50 (e.g., Yang et al. 2000, 2004, 2013). For particles with smaller scattering size parameters (1-50), so-called edge effects and wave interference have to be taken into account (Mitchell 2000; Yang et al. 2013), which lead to a general increase of the extinction coefficient up to about
3 with decreasing size parameter and to oscillations in the extinction coefficient in size parameter space of about $20 \%$ or smaller. For yet smaller size parameters $\left(\chi_{\text {scat }}<1\right)$, particles are comparable to or smaller than the wavelength and transition to the Rayleigh regime, associated with a decrease of extinction coefficient with decreasing size. Since the parameterizations presented in this paper are based on calculations that are based on the standard geometric optics approximations, these effects are neglected and $Q_{e}$ is assumed to be equal to 2 for all sizes. Thus, substantial errors in the extinction coefficient owing to the geometric optics approximation can be expected for small ice crystals. However, the contribution of these small crystals to the total extinction cross section is expected to be generally minor under many conditions, owing to their small projected area. To our best knowledge, a proper quantification of the errors in extinction cross sections and ice cloud radiation simulations resulting from the approximation $Q_{e}=2$ is not yet available. As discussed in section 2a, such an investigation is beyond the scope of the current paper and left for future work.

\section{c. Asymmetry parameter}

In the geometrics optics regime, the total scattering phase function $P_{\text {tot }}$ includes contributions from internal refraction and reflections $P_{\mathrm{RT}}$ (determined using ray tracing) and contributions from diffraction $P_{\text {dif }}$ (Macke et al. 1996):

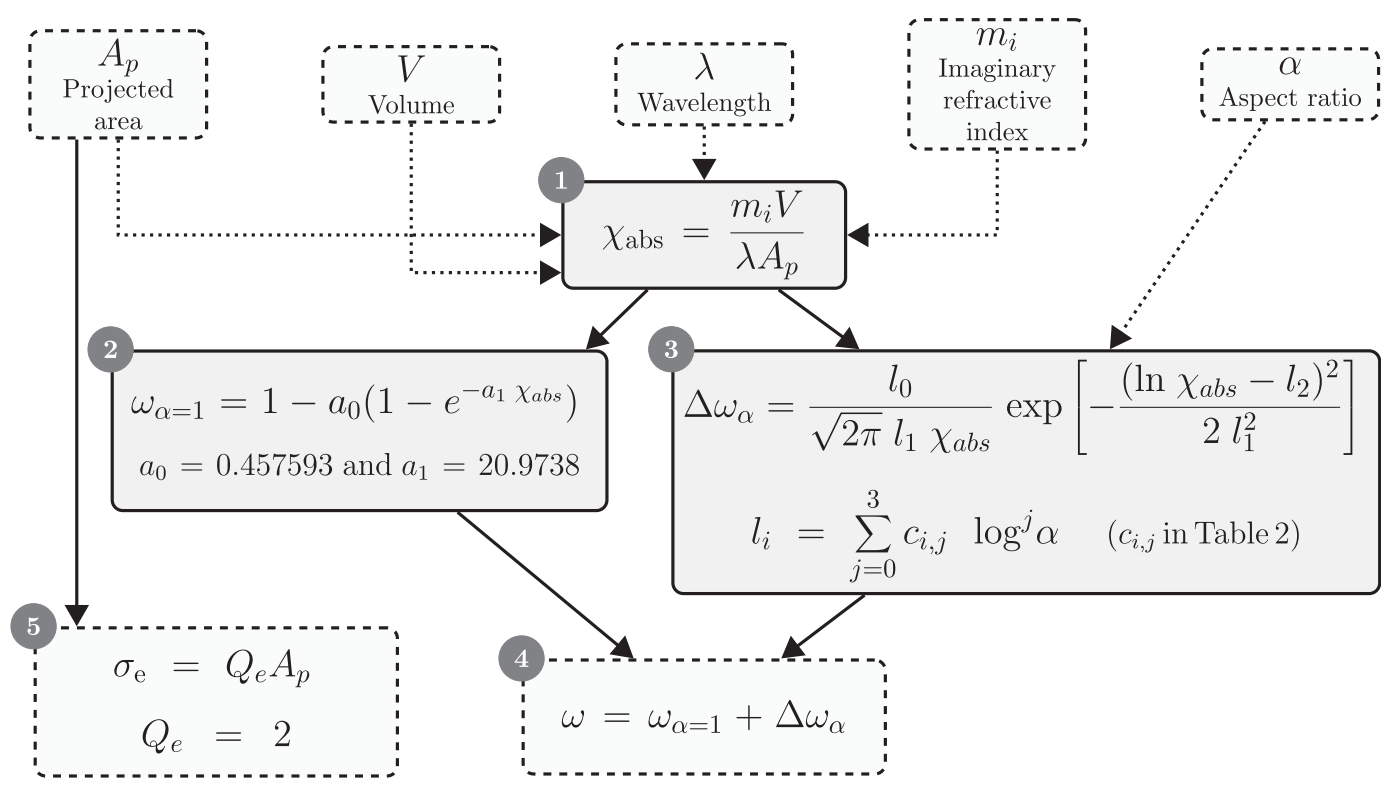

FIG. 4. Summary of the parameterization of single-scattering albedo (box 4), with the absorption size parameter in box 1, parameterization of single-scattering albedo for particles with aspect ratio of unity $\left(\omega_{\alpha=1}\right)$ in box 2 , and the parameterization of the relative difference between $\omega_{\alpha=1}$ and scattering albedo for particles with other aspect ratios in box 3. The geometric optics approximation for the extinction cross section is given in box 5 . 


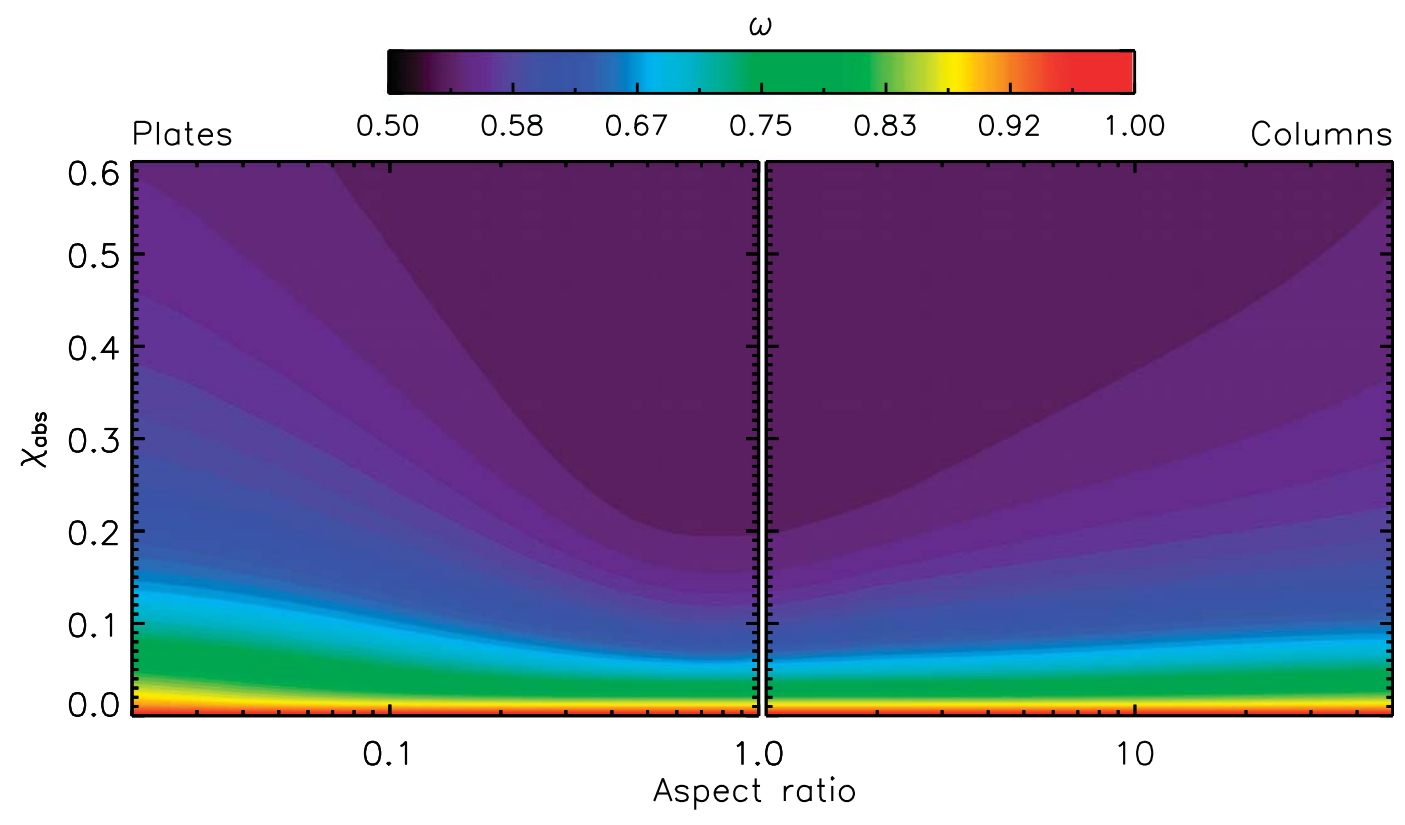

FIG. 5. Parameterized single-scattering albedo as a function of particle aspect ratio and absorption size parameter.

$$
P_{\mathrm{tot}}(\theta)=\frac{1}{2 \omega}\left[(2 \omega-1) P_{\mathrm{RT}}(\theta)+P_{\mathrm{dif}}(\theta)\right]
$$

The scattering phase function is often characterized by its first moment, the asymmetry parameter $g_{\text {tot }}$ (Macke et al. 1996):

$$
g_{\text {tot }}=\int_{0}^{\pi} P_{\text {tot }} \cos \theta \sin \theta d \theta=\frac{1}{2 \omega}\left[(2 \omega-1) g_{\mathrm{RT}}+g_{\mathrm{dif}}\right] .
$$

where $g_{\text {dif }}$ and $g_{\mathrm{RT}}$ are the asymmetry parameters of the diffractive contributions and the refractive plus reflective (or ray tracing) contributions to the phase function, respectively. A parameterization of $\omega$ is presented in the previous section. Note that at nonabsorbing wavelengths $(\omega=1)$, Eq. (12) reduces to

$$
g_{\text {tot }}=\frac{1}{2}\left(g_{\mathrm{RT}}+g_{\text {dif }}\right)
$$

Here we aim to parameterize $g_{\text {dif }}$ and $g_{\mathrm{RT}}$ as a function of particle volume, projected area, aspect ratio, distortion parameter, and wavelength. Then Eq. (12) can be used to obtain the total asymmetry parameter for any hexagonal ice crystal at any wavelength.

As shown in Fig. 6, the diffraction asymmetry parameter is mainly determined by the particle scattering size parameter $\chi_{\text {scat }}$. For $\chi_{\text {scat }}$ larger than about 100 , $g_{\text {dif }}=1$. For smaller $\chi_{\text {scat }}, g_{\text {dif }}$ can be calculated analytically with series of Bessel functions (van de Hulst 1957; Macke et al. 1996; Mishchenko and Macke 1998). Here, we simply approximate it with a parametric form justified by the integral over a diffraction pattern:

$$
g_{\text {dif }}=b_{0} e^{b_{1} \ln \chi_{\text {scat }}}+b_{2},
$$

with $b_{0}=-0.822315, b_{1}=-1.20125$, and $b_{2}=0.996653$, as shown in Fig. 6. The coefficients were obtained using a least squares fit. The diffraction asymmetry parameter

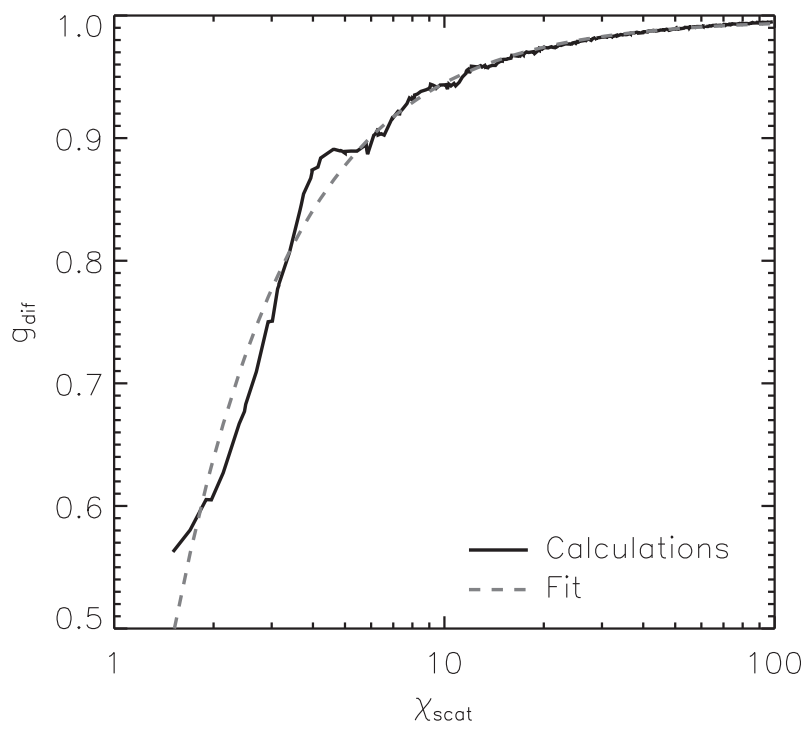

FIG. 6. Diffraction asymmetry parameter of hexagonal ice crystals with an aspect ratio of unity as a function of scattering size parameter. The gray dashed line represents the exponential fit described in the text. 


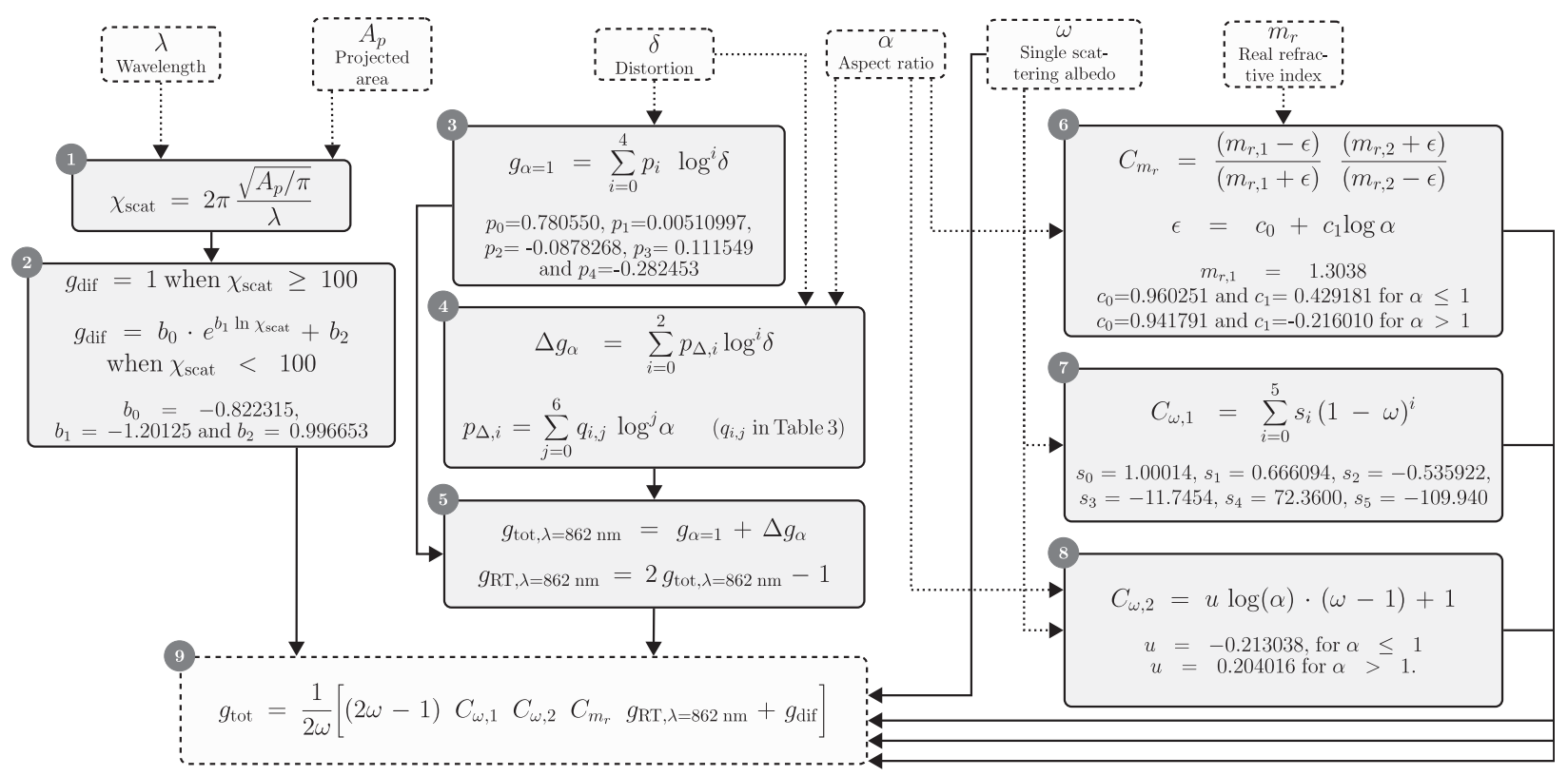

FIG. 7. Summary of the parameterization of asymmetry parameter $g_{\text {tot }}$ (box 9), with parameterization of the diffraction asymmetry parameter in boxes 1 and 2, parameterization of ray-tracing asymmetry parameter at $862 \mathrm{~nm}$ in boxes 3-5, and correction factors for real refractive index and absorption in boxes 6-8.

is limited to be greater than 0.5 . This parameterization of $g_{\text {dif }}$ is summarized in Fig. 7, boxes 1 and 2.

The refraction-plus-reflection asymmetry parameter depends on particle volume, projected area, aspect ratio, distortion parameter, and complex refractive index. However, at any given nonabsorbing wavelength, $g_{\text {RT }}$ of single hexagonal ice crystals mainly depends on aspect ratio and crystal distortion (cf. Fu 2007; Yang et al. 2008; Yang and Fu 2009; van Diedenhoven et al. 2012a). For example, Fig. 8 shows the total asymmetry parameter at a nonabsorbing wavelength of $\lambda=862 \mathrm{~nm}$ as a function of $\delta$ for several aspect ratios. Note that these calculations are made for large particles $\left(\chi_{\text {scat }} \gg 100\right)$, and thus $g_{\text {dif }}=1$ and the dependencies of the total asymmetry parameter on aspect ratio and distortion parameter seen in Fig. 8 are solely attributable to variations in $g_{\mathrm{RT}}$ [see Eq. (13)]. Here, the wavelength band at $862 \mathrm{~nm}$ is used as a reference because it corresponds to similar bands commonly used in aircraft and satellite retrievals of ice cloud properties (e.g., Chepfer et al. 2001; King et al. 2004; van Diedenhoven et al. 2012a,b). As described by van Diedenhoven et al. (2012a), sizes are varied so that the projected areas of the particles, assuming random orientation, correspond to the projected areas of spheres with radii of $14,20,28,40,56,80,113,160,226$, and $320 \mu \mathrm{m}$. The aspect ratio of columns is varied between 1 and 50 with 26 geometrically increasing steps. The aspect ratios of plates are the inverse of those for columns, for a total of 51 aspect ratios. The distortion parameter is varied between 0 and 0.8 in steps of 0.05 . Since GO calculations are independent of size for large particles, results are simply averaged over all sizes in order to decrease numerical noise. In the following, we aim to first parameterize the total asymmetry parameter at $\lambda=862 \mathrm{~nm}$ from which we derive $g_{\mathrm{RT}}$ at $\lambda=862 \mathrm{~nm}$ using Eq. (13). Subsequently, we derive factors to scale

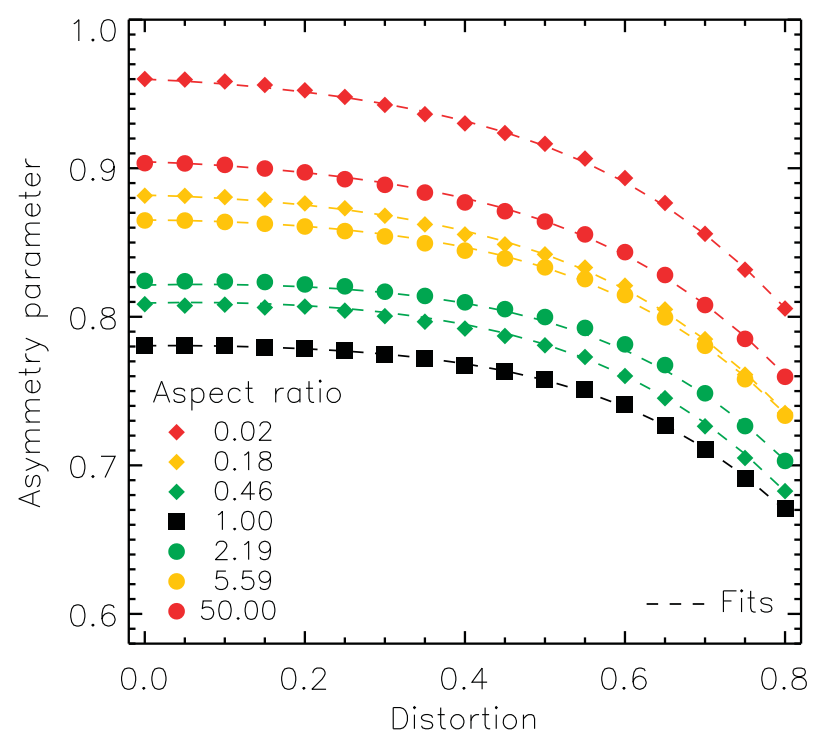

FIG. 8. Ice crystal total asymmetry parameters at $862 \mathrm{~nm}$ as a function of distortion parameter for various aspect ratios. Dashed lines represent the fits described in the text. 
the resulting $g_{\mathrm{RT}}$ to other wavelengths - that is, for other complex refractive indices.

Figure 8 shows that asymmetry parameters increase as aspect ratio departs from unity owing to the increase of parallel surface areas, leading to greater probability of light passing through the particle with low orders of refraction plus reflection and a minimal change of direction (Yang and Fu 2009). Increase of crystal distortion increases the chance of light refracted away from forward directions, leading to decreased asymmetry parameters (Yang et al. 2008).

Figure 8 shows that the dependence of $g_{\alpha=1}$ on $\delta$ for compact particles $(\alpha=1)$ can be approximated by a fourth-degree polynomial in $\delta$ with coefficients $p_{0}=$ $0.780550, p_{1}=0.00510997, p_{2}=-0.0878268, p_{3}=$ 0.111549 , and $p_{4}=-0.282453$. Similar to the procedure followed above in the parameterization of singlescattering albedo, the differences $\Delta g_{\alpha}$ between $g_{\alpha=1}$ and $g_{\text {tot }}$ at all other aspect ratios in our database are then fit with third-degree polynomials in $\delta$ with coefficients $p_{\Delta, i}$. In turn, the dependence of those $p_{\Delta, i}$ values on $\log (\alpha)$ is found to be well approximated by sixth-degree polynomial functions in $\alpha$ for both columns and plates. (The degrees of the polynomials are chosen to keep the maximum differences of parameterized and reference $g_{\text {tot }}$ values below 0.005.) The parameters for this fit are listed in Table 3, and the results are shown in Fig. 8. Thus, $\Delta g_{\alpha}$ is determined by first computing from $\alpha$ the $p_{\Delta, i}$ values, and subsequently computing from $\delta$ the $\Delta g_{\alpha}$ values for the required $\alpha-\delta$ combination. In turn, the total asymmetry parameter at $862 \mathrm{~nm}$ for the required values of aspect ratio and distortion is computed from

$$
g_{\text {tot }}(\alpha, \delta, \lambda=862 \mathrm{~nm})=g_{\alpha=1}(\delta)+\Delta g_{\alpha}(\alpha, \delta) .
$$

This parameterization of the total asymmetry parameter at $\lambda=862 \mathrm{~nm}$ is summarized in Fig. 7, boxes 3-5.

Figure 9 shows the parameterized $g_{\text {tot }}$ at $862 \mathrm{~nm}$ and the differences from the reference calculations (cf. Fig. 1 in van Diedenhoven et al. 2012a). The asymmetry parameter at $862 \mathrm{~nm}$ is seen to be approximated with an accuracy better than 0.004 . The refraction plus reflection asymmetry parameter at $862 \mathrm{~nm}$ can be derived from this parameterization using $g_{\mathrm{RT}}=2 g_{\text {tot }}-1$, since $g_{\text {dif }}=1$ and $\omega=1$ [see Eq. (13)]. Next, we will demonstrate how this parameterized asymmetry parameter for $\lambda=862 \mathrm{~nm}$ can be scaled to other wavelengths- that is, for other complex refractive indices.

The dependence of $g_{\mathrm{RT}}$ on the real part of the refractive index $m_{r}$ is investigated by performing geometric optics calculations for smooth large hexagonal crystals at the wavelength bands listed in Table 1, but artificially setting the imaginary part of the refractive
TABLE 3. Coefficients $q_{i, j}$ of the sixth-order polynomial to compute coefficients $p_{\Delta, i}$ in Eq. (15). See also Fig. 7, box 4 .

\begin{tabular}{lcccc}
\hline \hline & $j$ & $q_{0, j}$ & \multicolumn{1}{c}{$q_{1, j}$} & \multicolumn{1}{c}{$q_{2, j}$} \\
\hline Plates & 0 & -0.00133106 & -0.000782076 & 0.00205422 \\
& 1 & 0.0408343 & -0.00162734 & 0.0240927 \\
& 2 & 0.525289 & 0.418336 & -0.818352 \\
& 3 & 0.443151 & 1.53726 & -2.40399 \\
& 4 & 0.00852515 & 1.88625 & -2.64651 \\
& 5 & -0.123100 & 0.983854 & -1.29188 \\
& 6 & -0.0376917 & 0.187708 & -0.235359 \\
& & & & \\
& & & & \\
& & 0.00189096 & 0.000637430 & 0.00157383 \\
& 1 & 0.00981029 & 0.0409220 & 0.00908004 \\
& 2 & 0.732647 & 0.0539796 & -0.665773 \\
& 3 & -1.59927 & -0.500870 & 1.86375 \\
& 4 & 1.54047 & 0.692547 & -2.05390 \\
& 5 & -0.707187 & -0.374173 & 1.01287 \\
& 6 & 0.125276 & 0.0721572 & -0.186466 \\
\hline
\end{tabular}

index to zero (i.e., $\omega=1$ ). Figure 10 shows that $g_{\text {RT }}$ relative to its value at $862 \mathrm{~nm}\left(m_{r}=1.3038\right)$ is a smooth function of $m_{r}$ for a given aspect ratio. We hypothesize that, as Fresnel reflection on the particle surfaces is increased with an increasing refractive index, forward scattering is reduced and backward scattering is increased (i.e., asymmetry parameter is lowered). It is seen in Fig. 10 that the ratio of the asymmetry parameter with a certain refractive index $m_{r, 1}$ to that with another refractive index $m_{r, 2}$ is reasonably well approximated by functions of the form

$$
C_{m_{r}}=\frac{\left(m_{r, 1}-\epsilon\right)}{\left(m_{r, 1}+\epsilon\right)} \frac{\left(m_{r, 2}+\epsilon\right)}{\left(m_{r, 2}-\epsilon\right)},
$$

where $\epsilon$ is a fitting coefficient that depends on aspect ratio. Since we use calculations at $862 \mathrm{~nm}$ as a referencehere $m_{r, 1}=1.3038$. The functional form of Eq. (16) is inspired by the Fresnel equation for reflection (Born and Wolf 1999). The parameter $\epsilon$ is computed from the value that minimizes the root-mean-square difference between Eq. (16) and $g_{\mathrm{RT}}\left(m_{r, 2}\right)$ over the range of $m_{r, 2}$ in Table 1. The value of $\epsilon$ is found to be 0.960251 for compact ice crystals $(\alpha=1)$, and it decreases as the aspect ratio increasingly deviates from unity. We find that $\epsilon$ scales well with $\log (\alpha)$; that is,

$$
\epsilon(\alpha)=c_{0}+c_{1} \log \alpha,
$$

with $c_{0}=0.960251$ and $c_{1}=0.429181$ for plates and $c_{0}=$ 0.941791 and $c_{1}=-0.216010$ for columns. Essentially the same fit parameters are found when taking other $m_{r, 1}$ values and for particles with increased $\delta$ (not shown). This parameterization of dependence of $g_{\mathrm{RT}}$ on $m_{r}$ is summarized in Fig. 7, box 6. 

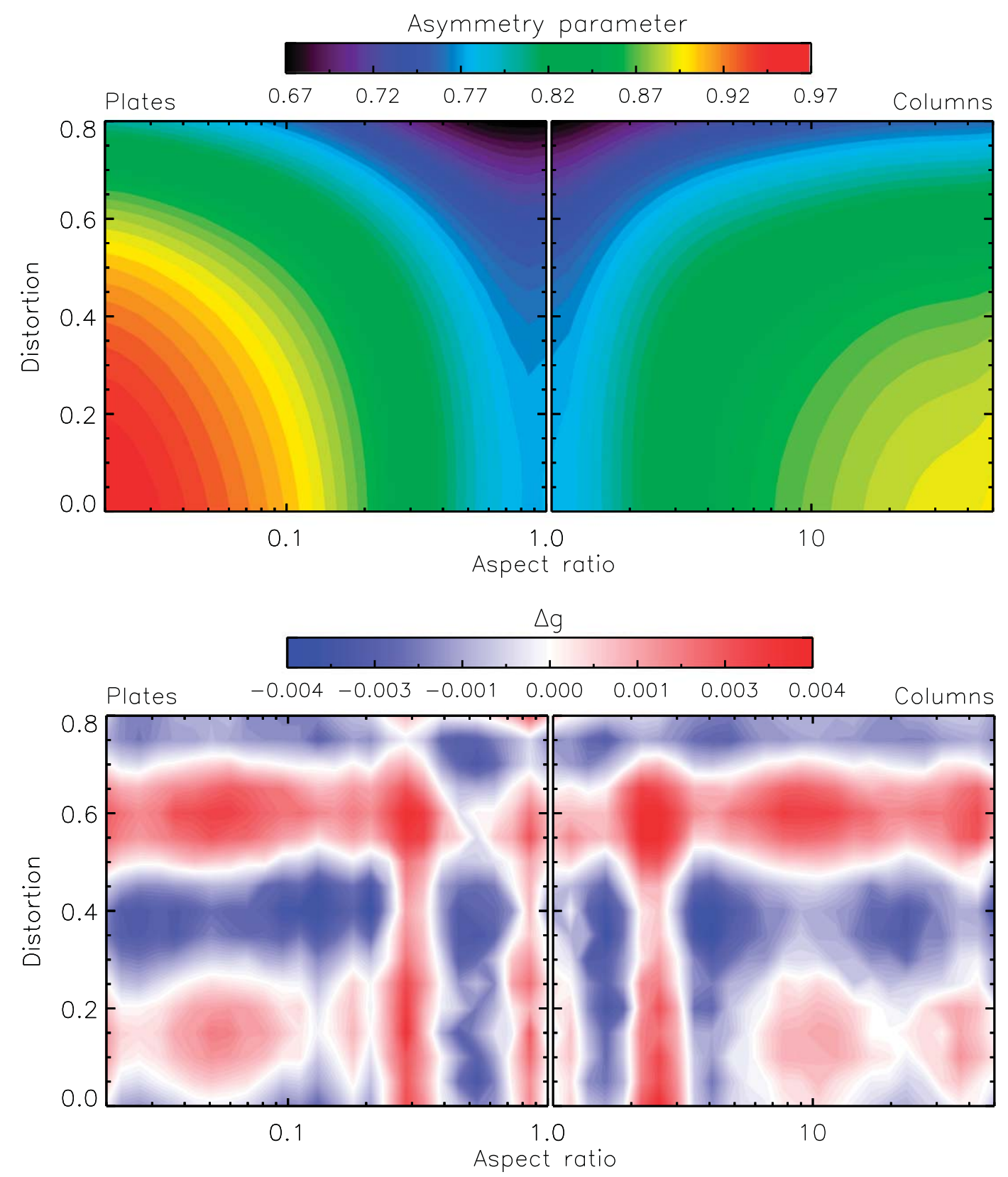

FIG. 9. (top) Parameterized total asymmetry parameters at $862 \mathrm{~nm}$ and (bottom) the differences from the reference calculations.

As noted by Fu (2007), Eq. (12) shows that the dependence of the total asymmetry parameter on singlescattering albedo is largely determined by the weighting of the ray-tracing and diffractive parts of the asymmetry parameter. However, $g_{\mathrm{RT}}$ itself also depends on the single-scattering albedo; ignoring this dependence can lead to errors in the total asymmetry parameter up to about $5 \%$, depending on the wavelength band and aspect ratio, as shown in Fig. 11a. This effect was neglected by Fu (2007). To investigate the dependence of $g_{\mathrm{RT}}$ on absorption, Fig. 11b shows $g_{\text {RT }}$ relative to its value when $\omega=1$ as a function of coalbedo $(1-\omega)$. The value of $g_{\mathrm{RT}}$ is found to increase nearly linearly with $(1-\omega)$ for $(1-\omega) \lesssim 0.3$, but to decrease for greater $(1-\omega)$. This dependence of $g_{\mathrm{RT}}$ on $\omega$ is seen to vary with aspect ratio. The dependence also varies with the real part of the refractive index, as evident from the differences between wavelengths above and below $3 \mu \mathrm{m}$. The dependence is not found to be substantially affected by increasing distortion (not shown). We hypothesize that 


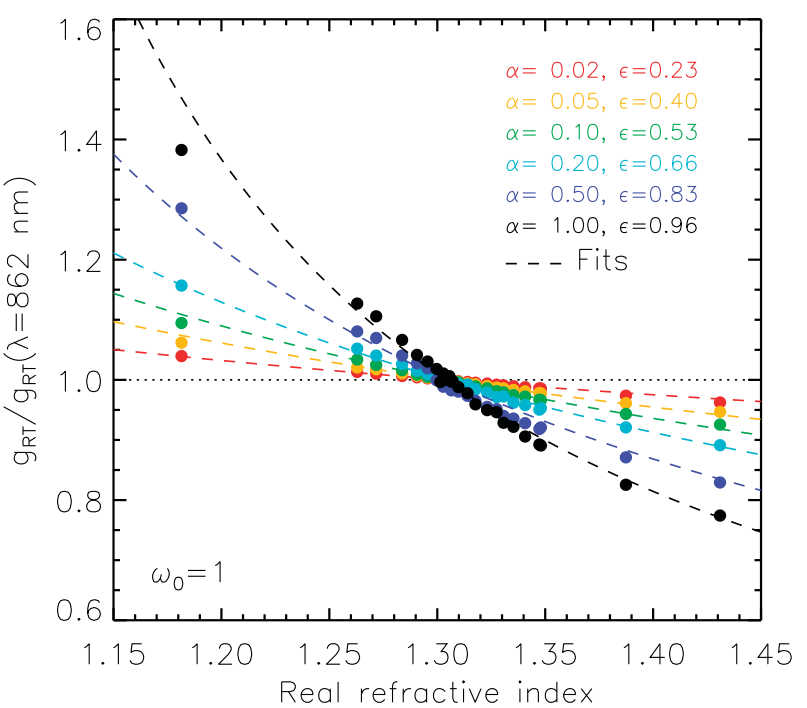

FIG. 10. The refraction-plus-reflection asymmetry parameter of nonabsorbing ice crystals as a function of real refractive index, relative to its value at $862 \mathrm{~nm}$, or $m_{r}=1.3038$. Calculations for several plate aspect ratios are indicated by color. Dashed lines represent fits as described in the text with indicated fit parameters $\epsilon$.

the dependence of $g_{\mathrm{RT}}$ on $\omega$ results from two competing effects with increasing absorption: 1) contributions of shorter paths through the particle with lower orders of scattering, which generally are directed forward (Yang and $\mathrm{Fu} 2009$ ), are increased, tending to increase $g_{\mathrm{RT}}$, and 2) contributions of rays reflected sideways or backward on the particle surface (i.e., external reflections) are increased relative to the rays entering and exiting the particle, tending to decrease $g_{\text {RT }}$. To determine a factor to partly correct the refraction plus reflection asymmetry parameter that was parameterized above assuming no absorption [i.e., $g_{\mathrm{RT}}(\omega=1)$ ] for variations in coalbedo, we first fit a fifth-degree polynomial in $(\omega-1)$ through the calculated $g_{\mathrm{RT}} / g_{\mathrm{RT}}(\omega=1)$ values for $\alpha=1$ shown in Fig. 11b. For simplicity, here we do not account for the dependence of $g_{\text {RT }}$ on real refractive index. The six coefficients $s_{i}$ for this fitted correction factor $C_{\omega, 1}$ are $1.00014,0.666094,-0.535922,-11.7454,72.3600$, and -109.940 . Next, the relative differences (denoted $C_{\omega, 2}$ ) between $C_{\omega, 1}$ and calculated values of $g_{\mathrm{RT}} / g_{\mathrm{RT}}(\omega=1)$ for aspect ratios deviating from unity are determined and fit by the linear function $C_{\omega, 2}=k(\omega-1)+1$, where $k$ is a fit parameter that depends on aspect ratio. Analogous to the procedure used above, we finally fit the dependence of $k$ on aspect ratio using the linear functional form $k=u \log (\alpha)$. Thus, $C_{\omega, 2}$ is computed by

$$
C_{\omega, 2}=u \log (\alpha)(\omega-1)+1 \text {, }
$$

where $u=-0.213038$ for plates and $u=0.204016$ for columns. For illustration, the fit result for $\alpha=0.1$ is also

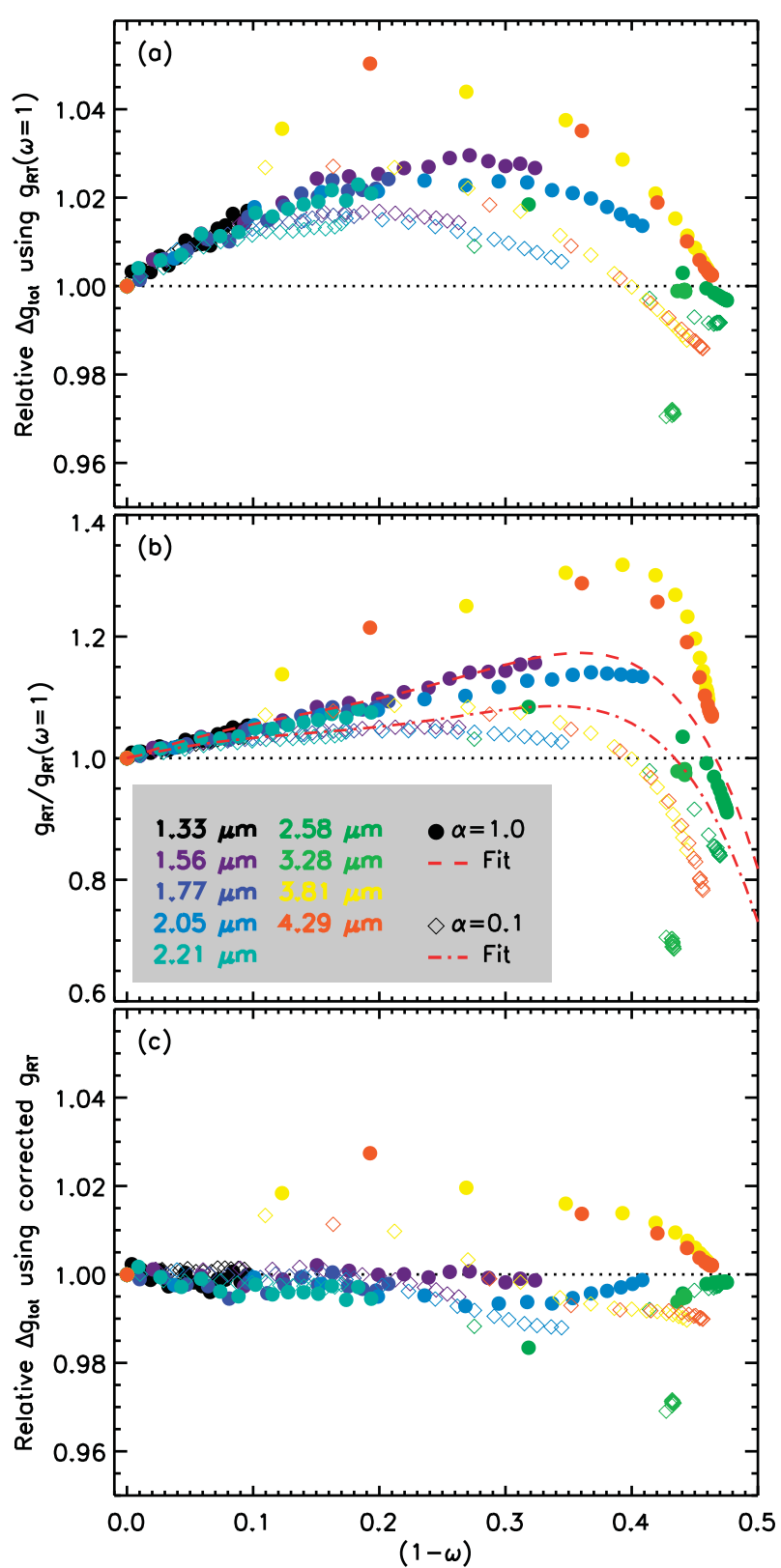

FIG. 11. (a) Relative differences between calculated total asymmetry parameters and those estimated by using Eq. (12) and the refraction-plus-reflection asymmetry parameter for nonabsorbing particles $(\omega=1)$. Calculations for different wavelength bands are indicated by colors. Filled circles represent compact particles $(\alpha=1)$ and open diamonds indicate thin plates $(\alpha=0.1)$. Only two aspect ratios are shown to reduce clutter. (b) Relative differences in the refraction-plus-reflection asymmetry parameter for particles with $\omega=1$ and values for particles with lower singlescattering albedos. Red lines indicate fits for $\alpha=1$ (dashed) and $\alpha=0.1$ (dashed-dotted) as described in the text. (c) As in (a), but using the derived factors as described in the text to correct the refraction-plus-reflection asymmetry parameter for absorption effects. 


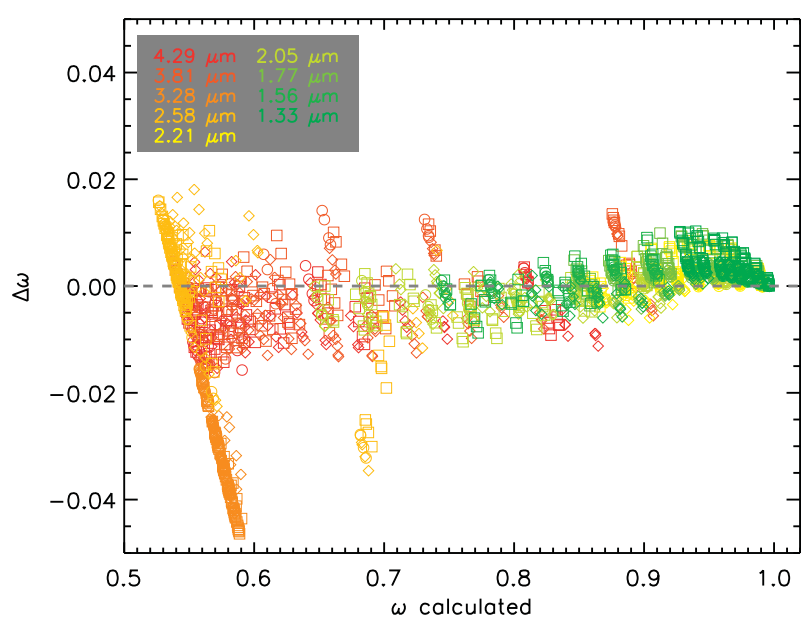

FIG. 12. Absolute difference between parameterized and calculated single-scattering albedos for several wavelength bands as indicated by colors. Circles, diamonds, and squares indicate hexagonal columns, plates, and compact hexagons with aspect ratio of unity, respectively.

shown in Fig. 11b. The computation of these factors to partly correct $g_{\mathrm{RT}}(\omega=1)$ for variations in coalbedo is summarized in Fig. 7, boxes 7 and 8. Figure 11c shows the difference between the calculated $g_{\text {tot }}$ values and those parameterized using $g_{\mathrm{RT}}(\omega=1)$ with correction factors $C_{\omega, 1}$ and $C_{\omega, 2}$. The errors are generally below $1 \%$ for $\lambda<2.2 \mu \mathrm{m}$ and below $2 \%$ for longer wavelengths.

Finally, the asymmetry parameter for hexagonal crystals can be approximated via

$$
\begin{aligned}
& g_{\text {tot }}\left(\alpha, \delta, m_{r}, \omega\right) \\
& \quad=\frac{1}{2 \omega}\left[(2 \omega-1) C_{\omega, 1} C_{\omega, 2} C_{m} g_{\mathrm{RT}, \lambda=862 \mathrm{~nm}}+g_{\mathrm{dif}}\right],
\end{aligned}
$$

as summarized in Fig. 7, box 9. The single-scattering albedo $\omega$ is parameterized as described in section $2 \mathrm{~b}$ and Fig. 4.

\section{Results}

\section{a. Optical properties of individual particles}

The parameterization is tested on GO calculations for hexagonal crystals with aspect ratios varying between $0.05,0.1,0.5,1,2,5$, and 20; effective distances of 10, 30, $50,70,90,110,150$, and $190 \mu \mathrm{m}$; and distortion parameters of $0,0.2,0.4$, and 0.6 and at wavelength bands listed in Table 1. For all wavelength bands with substantial absorption, absolute differences between calculated and parameterized single-scattering albedos are generally below 0.015 , as seen in Fig. 12. Only for bands with $m_{i}>0.02$

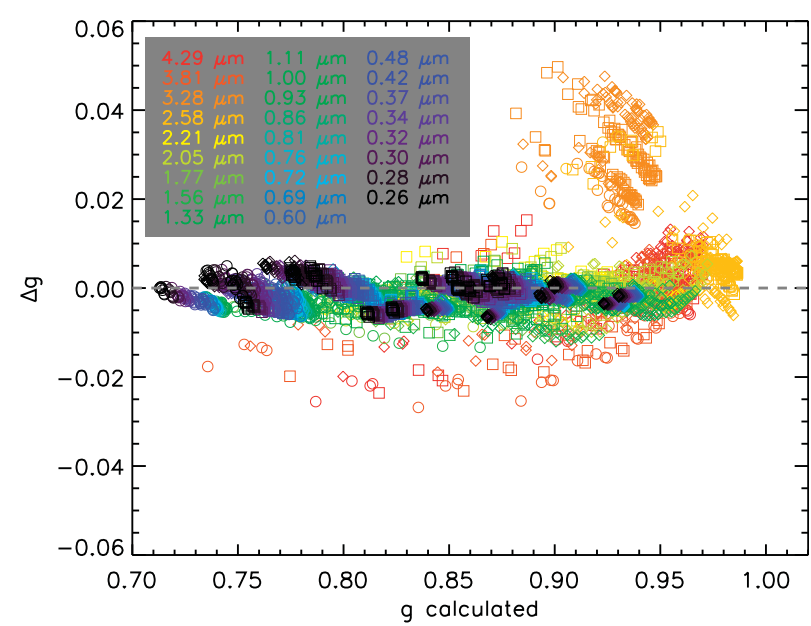

FIG. 13. As in Fig. 12, but for asymmetry parameter.

(at 2.6 and $3.3 \mu \mathrm{m}$ ) are absolute differences up to 0.05 obtained, especially for small single-scattering albedos, which is expected considering the different dependence of $\omega$ on $\chi_{\text {abs }}$ for these bands seen in Fig. 1. Though not evident in Fig. 12, the smallest errors are obtained for relatively compact particles with $0.5 \leq \alpha \leq 2$.

Figure 13 shows the absolute differences between calculated and parameterized asymmetry parameters for all wavelength bands listed in Table 1. For nonabsorbing bands, the accuracy of the parameterization is better than 0.01 . For moderately absorbing wavelengths, absolute differences between calculated and parameterized asymmetry parameters do not exceed 0.015 , but the parameterization is slightly biased toward underestimating the asymmetry parameter, especially for larger, more absorbing particles. For strongly absorbing wavelengths $\left(m_{i}>0.02\right)$, errors in the parameterized asymmetry parameter up to 0.05 are seen, which is attributable to the deviations of single-scattering albedo from the parameterization for these bands, and the fact that the correction of $g_{\mathrm{RT}}$ for its dependence on the single-scattering albedo for these bands are not well represented by the fits (Fig. 11). The smallest errors are again obtained for relatively compact particles with $0.5 \leq \alpha \leq 2$.

\section{b. Application to flux calculations}

To assess the errors on flux in heating calculations caused by errors in parameterized optical properties, we apply two-stream calculations (see appendix B; Coakley and Chylek 1975; Wiscombe and Grams 1976) to uniform ice cloud layers. We use the same optical properties of single ice particles throughout. To obtain optical properties for realistic ice size distributions, gamma size distributions of the form 

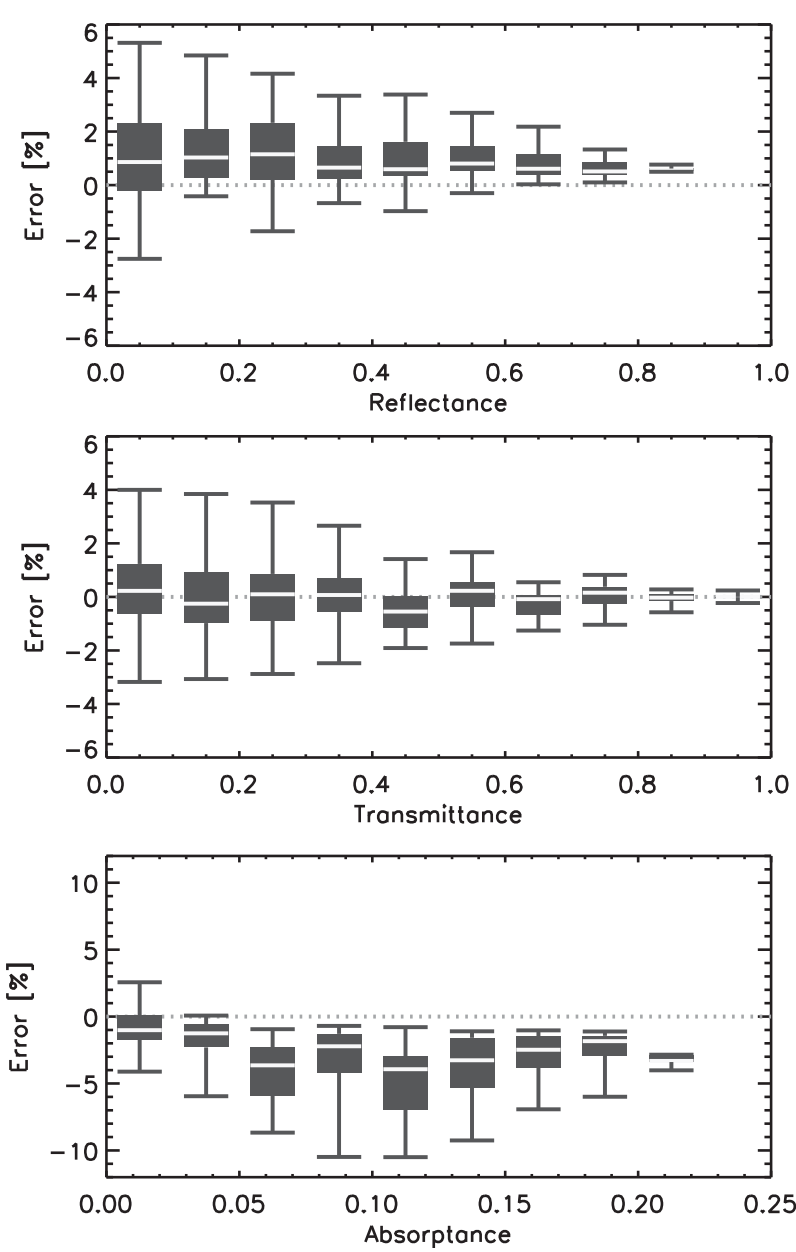

FIG. 14. Relative parameterization errors in transmittance, reflectance, and absorptance of uniform cloud layers. The box-andwhisker plots show median values, 25 th and 75 th percentiles, and full ranges of the errors in several transmittance, reflectance, and absorptance bins. Cloud optical thicknesses range from 0.01 to 100 and wide ranges of different size distributions, aspect ratios, and distortion parameters are included, as described in the text. A solar zenith angle of $60^{\circ}$ is assumed. Further details provided in text.

$$
\frac{d N}{d D}(D)=N_{0} D^{\mu_{p}} e^{-\lambda_{p} D}
$$

are applied (Mitchell 1991; Heymsfield et al. 2002; Baum et al. 2005b), where $D$ is the maximum particle dimension and $\mu_{p}$ and $\lambda_{p}$ are the shape and slope parameters of the size distribution. Here $N_{0}$ is a normalization factor. A range of size distributions is applied by varying $\lambda_{p}$ in Eq. (20) from 20 to $200 \mathrm{~cm}^{-1}$ with increments of $20 \mathrm{~cm}^{-1}$ and using the relation between $\mu_{p}$ and $\lambda_{p}$ determined by Heymsfield et al. (2002) from in situ measured ice size distributions; namely, $\mu_{p}=\lambda_{p}^{0.64}-2$ (with $\lambda_{p}$ in units of per centimeter). [We note that there is ample uncertainty in this relation because of unknown shattering issues in these measurements (Korolev et al. 2011), but our results are largely insensitive to this assumption.] Two-stream calculations are made for 10 different optical thicknesses ranging from 0.01 to 100 and a solar zenith angle of $60^{\circ}$. Spectral reflectance and transmittance calculations are made for the wavelength bands listed in Table 1. Relative solar reflectance $R$ and transmittance $T$ are obtained through integration of the spectral quantities, weighted by the relative contribution to incoming spectral solar flux by each wavelength band (cf. Fu 1996). Absorptance is then defined as $1-R-T$.

Figure 14 shows errors in reflectance, transmittance, and absorptance resulting from the parameterization. Relative errors in reflectance generally increase with decreasing reflectance and the same is generally true for the error in transmittance. The error in absorptance peaks around absorptance values of 0.1 , which occur mostly at optical thicknesses of 20-60. Errors in reflectances are mostly caused by errors in the parameterization of asymmetry parameter, while errors in transmittance and absorptance are mostly affected by parameterization errors in the single-scattering albedo, as might be expected. Overall median errors and interquartile ranges in reflectance, transmittance, and absorptance are listed in Table 4 for several aspect ratio ranges. Lowest errors and interquartile ranges are generally obtained for relatively compact crystals $(0.5 \leq$ $\alpha \leq 2$ ), while more extreme aspect ratios yield somewhat larger errors. The overall relative root-mean-squared errors in reflectance, transmittance, and absorptance are $1.4 \%, 1.1 \%$, and $3.4 \%$, respectively. Crystal distortion is found to have no significant effect on the resulting errors (not shown). Similar errors are also obtained for other solar zenith angles (not shown; cf. Fu 1996).

To put our results in perspective with those of other parameterizations, we note that the averaged absolute values of the relative errors in reflectance, transmittance, and absorptance of $1.1 \%, 0.7 \%$, and $2.5 \%$, respectively,

TABLE 4. Medians and interquartile ranges (within parentheses) of relative errors in fluxes and absorptance owing to the proposed parameterization for several aspect ratio ranges. All values are percentages.

\begin{tabular}{lcccc}
\hline \multicolumn{1}{c}{ Quantity } & All $\alpha$ & $0.5 \leq \alpha \leq 2$ & $\alpha \leq 0.1$ & $\alpha \geq 10$ \\
\hline Reflectance & $0.69(1.1)$ & $0.50(0.52)$ & $1.4(2.1)$ & $0.85(1.0)$ \\
Transmittance & $0.00(0.74)$ & $0.00(0.49)$ & $-0.07(1.26)$ & $0.09(1.15)$ \\
Absorptance & $-1.9(2.2)$ & $-1.4(0.61)$ & $-2.7(1.7)$ & $-4.3(3.3)$ \\
\hline
\end{tabular}


resulting from our parameterization, are comparable to the corresponding values obtained by the parameterization of $\mathrm{Fu}$ (1996), which are $1.2 \%, 0.3 \%$, and $2.9 \%$, respectively, for a solar zenith angle of $60^{\circ}$ (their Fig. 6). The absolute maximum errors in reflectance, transmittance, and absorptance of $6 \%, 4 \%$, and $11 \%$, respectively, are also comparable or somewhat larger than the corresponding maximum errors reported by $\mathrm{Fu}(1996)$, which are about $6 \%, 2 \%$, and $8 \%$. However, the parameterization scheme presented here is evaluated over a much wider range of particle size distributions, cloud optical thicknesses, aspect ratios, and distortion values than the scheme by Fu (1996). Moreover, our parameterization scheme is much more flexible than previous schemes.

Finally, we note that the errors given here do not include any possible errors owing to the application of the standard geometric optics approximations - the most important being the approximation $Q_{e}=2$. As stated above, quantification of such errors is currently lacking and left for future work.

\section{Practical application in atmospheric models}

It can now be considered conventional wisdom that shortwave optical properties of complex aggregates of hexagonal ice crystals at a given wavelength are mainly determined by their effective distance, distortion, and the aspect ratios of their components (e.g., Fu 1996, 2007; Um and McFarquhar 2007, 2009; van Diedenhoven et al. 2012a). Although the parameterization proposed here is based on single hexagonal plates and columns, it can provide optical properties of particles with any combination of volume, projected area, aspect ratio, and distortion parameter. In the case of complex aggregates, such as bullet rosettes and aggregates of columns or plates, the aspect ratio to be used in the parameterization is the mean aspect ratio of the components of the complex particle, as described by $\mathrm{Fu}$ (2007). For example, in the case of bullet rosettes, which are commonly defined as a structure of a number of bullets (usually six) that all have the same aspect ratio (Iaquinta et al. 1995; Yang and Liou 1998; Garrett et al. 2001), the aspect ratio to be used in the parameterization is the aspect ratio of the individual bullets, while the volume and projected area are quantities determined for the whole rosette. These assumptions are essentially the same as those made by the parameterization by $\mathrm{Fu}(1996,2007)$.

The flexibility of our parameterization can be exploited to efficiently obtain ice optical properties that are consistent with microphysical assumptions made in a cloud or climate model. Models of cloud microphysics commonly use power laws to relate ice crystal mass $m$ to crystal maximum dimension $D$ :

$$
m=a_{m} D^{b_{m}}
$$

Habit-dependent power laws relating particle projected area to maximum dimension are also available in the literature (e.g., Mitchell et al. 1996; Baker and Lawson 2006):

$$
\begin{aligned}
A_{p} & =a_{A} D^{b_{A}} \\
& =a_{A}\left(\frac{m}{a_{m}}\right)^{b_{A} / b_{m}} .
\end{aligned}
$$

Furthermore power-law relationships are available that relate particle aspect ratio to maximum dimensions (e.g., Auer and Veal 1970; Um and McFarquhar 2007, 2009) that can be used in treatments of cloud microphysics that require assumptions about particle aspect ratio (e.g., Böhm 1989, 1992; Wood et al. 2001; Westbrook et al. 2008; Westbrook 2008).

The parameterization presented here can be used to compute the ice crystal shortwave optical properties that are consistent with the assumed (or predicted) particle mass, area, and aspect ratios. Crystal distortion is the only parameter important for ice shortwave optical properties that is not considered to affect ice microphysics. Although the parameterization is designed to be combined with microphysical schemes that use assumptions about particle mass, area, and aspect ratio, in the case of microphysics parameterizations that do not include assumptions about area and/or aspect ratio, reasonable assumptions for those quantities can be made that are consistent with the assumed mass-dimension relations and observations (e.g., Mitchell et al. 1996; Baker and Lawson 2006; Auer and Veal 1970; Um and McFarquhar 2007, 2009).

The parameterization described in this paper yields the shortwave optical properties of individual ice crystals that need to be integrated over size distributions. Such an integration can be efficiently performed by binning the size distribution (Baum et al. 2005b). Since the parameterization can yield optical properties for any particle size, an unlimited number of bins can be used for this numerical integration, although Liu et al. (2012) showed that using about 40 bins is generally sufficient.

To illustrate such an application of the parameterization, we calculate fluxes for a uniform cloud layer consisting of ice crystals with varying aspect ratios, distortion parameters, and projected-area and mass relationships. First, we assume mass-dimension and projected 
area-dimension relationships consistent with aggregates of side planes as given by Mitchell et al. (1996); that is, $a_{m}=0.0033 \mathrm{~g} \mathrm{~cm}^{-b_{m}}, b_{m}=2.2, a_{A}=0.2285 \mathrm{~cm}^{2-b_{A}}$, and $b_{A}=1.88$ (such relations are traditionally in cgs units). Ice crystal maximum dimension is varied from 1 to $1000 \mu \mathrm{m}$ in $1-\mu \mathrm{m}$-wide bins. Note that the power-law parameters for aggregates of side planes as given by Mitchell et al. (1996) were defined for maximum dimension larger than $600 \mu \mathrm{m}$. However, for this illustration we apply them to all sizes, but limit the mass and the ratio of mass and area not to exceed those for a solid ice sphere. Furthermore, we consider sizeindependent aspect ratios varying from 0.02 to 50 , and size-independent distortion parameters varying from 0 to 0.8 . Optical properties are calculated using the parameterization for all wavelength bands listed in Table 1 and are subsequently integrated over a size distribution given by Eq. (20) with $\mu_{p}=1.5$ and $\lambda_{p}=$ $100 \mathrm{~cm}^{-1}$ (Heymsfield et al. 2002). The resulting bulk effective diameter [Eq. (5)] is $84 \mu \mathrm{m}$, independent of aspect ratio. Note that for some combinations of wavelength, maximum dimension, and aspect ratio, the particle size parameters may be smaller than the lower limit generally accepted for the geometric optics approximations applied. However, as discussed above, quantification of the resulting errors is currently not available. Two-stream calculations of cloud reflectance and transmittance are made as described above (see also appendix B) for a cloud layer with an optical thickness of 4 . The cloud layer is embedded between 10 and $11 \mathrm{~km}$ in a tropical atmosphere based on conditions observed during the Tropical Warm Pool International Cloud Experiment (TWP-ICE) campaign near Darwin, Northern Territory, Australia (Fridlind et al. 2012). For simplicity, the surface is assumed to be black. The results are spectrally integrated to obtain solar upwelling fluxes at the top of atmosphere and downwelling fluxes at the surface. As shown in Figs. 15a and 15c, the variation of radiative fluxes with aspect ratio and crystal distortion strongly echo the dependence of the asymmetry parameter on these parameters seen in Fig. 9 (top). Furthermore, the cloud reflectance and transmittance are almost exactly anticorrelated (not shown) leading to a cloud absorptance that is effectively independent of aspect ratio and crystal distortion (Fig. 15e).

Second, to illustrate the additional dependence of radiative fluxes and cloud absorptance on projectedarea and mass relationships, we artificially scale the projected area of the ice crystals to obtain bulk effective diameters between 25 and 200. [Essentially the same results are obtained by scaling masses as apparent from Eqs. (5), (21), and (22).] Figures 15b and 15d show the dependence of radiative fluxes on effective diameter and aspect ratio for smooth crystals $(\delta=0)$. The dependence of the fluxes on effective diameter is seen to be weaker than the dependence on aspect ratio. Cloud absorptance, however, is strongly dependent on effective diameter but only weakly dependent on aspect ratio (Fig. 15f), as might be expected. Note that the dependence of absorptance on effective diameter and aspect ratio resembles the variation of single-scattering albedo seen in Fig. 5. Similar dependencies are obtained for crystals with greater distortion $(\delta>0)$.

The example seen in Fig. 15 illustrates that ice crystal volume, projected area, aspect ratio, and distortion parameter that are inconsistent with a model's microphysics can yield substantial errors in radiative fluxes and absorptance (cf. Baran 2012). Our parameterization provides for a consistent treatment that avoids such errors. Our approach uses the geometric optics approximations, which are less applicable for longwave radiation calculations. However, longwave radiation is mainly determined by absorption, which in turn is determined by particle volume and projected area, largely independent of particle shape (Fu et al. 1999). For longwave flux calculations, optical properties that are largely consistent with the shortwave optical properties obtained with our parameterization may be calculated by applying Lorentz-Mie theory to collections of spheres that conserve ice crystal volume and projected area (Grenfell and Warren 1999; Fu et al. 1999; Neshyba et al. 2003), although such an approach may lead to errors in cloud emissivity of order $10 \%$ mainly attributable to the overestimation of tunneling effects (Fu et al. 1999; Mitchell et al. 2006). The modified anomalous diffraction approximation (MADA; Mitchell et al. 2006) partly accounts for tunneling effects using shape-dependent tunneling efficiency factors.

\section{Conclusions}

The parameterization scheme for optical properties of individual ice crystals presented in this paper is based on the same principles as the well-known scheme developed by Fu (1996, 2007), namely, that optical properties of complex, aggregated ice crystals can be approximated by those of single hexagonal crystals with varying size, aspect ratio, and distortion parameter. For example, in the case of a bullet rosette, the aspect ratio used in such a parameterization is the aspect ratio of the individual bullets, while the volume and projected area are the quantities determined for the whole rosette. Previous schemes by Fu $(1996,2007)$ and others are restricted to preselected wavelength bands, particle geometries, and distortion levels. Our scheme further extends these concepts to provide single-scattering albedos and asymmetry 

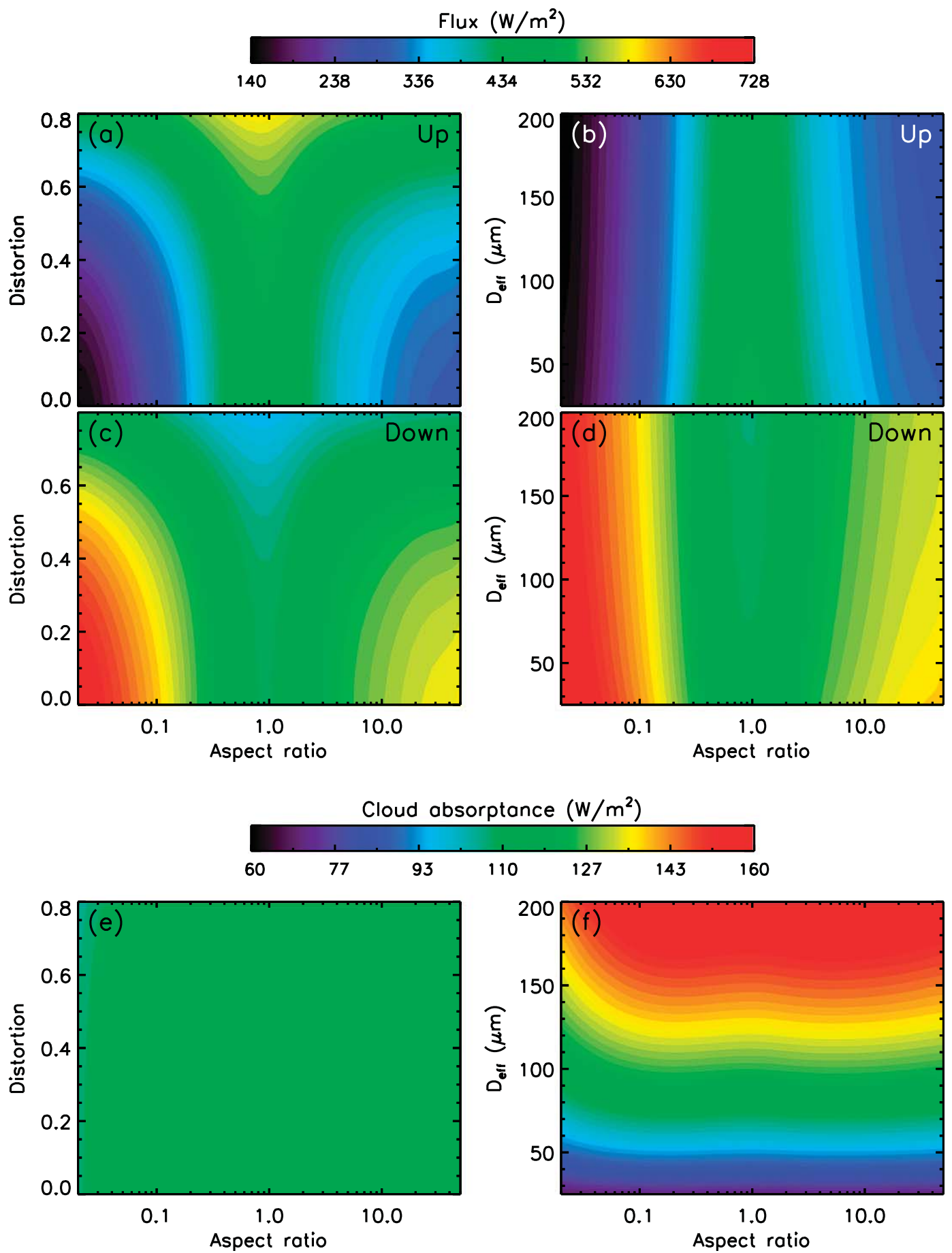

FIG. 15. Illustration of variations of (a),(b) upwelling- and (c),(d) downwelling fluxes and (e),(f) cloud absorptance (left) as a function of aspect ratio and distortion and (right) as a function of aspect ratio and effective diameter. The cloud layer has an optical thickness of 4 and is embedded in a tropical atmosphere between 10 and $11 \mathrm{~km}$. The solar zenith angle is $60^{\circ}$.

parameters for individual ice crystals with any combination of volume, projected area, aspect ratio (of particle components), and distortion parameter and at any wavelength in the shortwave.
Similar to the scheme developed by Fu $(1996,2007)$, the parameterization of single-scattering albedo and asymmetry parameter is based on geometric optics calculations for single hexagonal crystals with varying size, 
aspect ratio, and crystal distortion. In the standard geometric optics implementation used here, the extinction cross section is simply twice the projected area of crystals in random orientation. The ice crystal single-scattering albedo is parameterized in terms of particle aspect ratio and an absorption size parameter that is proportional to the ratio of the imaginary part of the refractive index to the wavelength multiplied by the ratio of particle volume to projected area. The parameterization of singlescattering albedo uses 26 coefficients. The asymmetry parameter of hexagonal ice crystals is parameterized in terms of particle aspect ratio, crystal distortion, projected area, single-scattering albedo, and wavelength, using 62 coefficients in total.

The scheme is tested for a large variety of hexagonal crystals in several wavelength bands from 0.2 to $4 \mu \mathrm{m}$, revealing absolute errors in both single-scattering albedo and asymmetry parameter that are generally below 0.015. Furthermore, using a large variety of particle aspect ratios, size distributions, and cloud optical thicknesses, the relative root-mean-square errors in cloud reflectance, transmittance, and absorptance are shown to be $1.4 \%, 1.1 \%$, and $3.4 \%$, respectively. Note, however, that these errors given do not include any possible errors owing to the application of the standard geometric optics approximations, as a quantification of such errors is currently lacking and left for future work.

As we demonstrate, this parameterization scheme is flexible enough to obtain ice crystal optical properties that are consistent with any assumptions about ice crystal mass (equivalent to bulk volume), projected area, and aspect ratio, which might be used in modern ice microphysics schemes to compute fall speeds and depositional growth rates of ice particles. The scheme can also be used to estimate optical properties of ice crystals of which the mass, projected area, and component aspect ratio are derived from measurements of in situ probes or remote sensing (e.g., Mitchell et al. 1996; Korolev and Isaac 2003; Um and McFarquhar 2007; Lawson et al. 2010; Mauno et al. 2011; Lindqvist et al. 2012; van Diedenhoven et al. 2012a, 2013). Appropriate distortion parameter values can be derived from satellite and aircraft remote sensing measurements (van Diedenhoven et al. 2012a,b, 2013).

A Python computer code is provided as supplemental material.

Acknowledgments. This material is based upon work supported by the NASA ROSES program under Grant NNX11AG81G. We are grateful for the contributions from three anonymous reviewers. We thank William Martin for his help in coding the Python program. We would like to thank Dr. Petri Räisänen for reporting a typographical error in Eq. (8) in the early online release of this paper.

\section{APPENDIX A}

\section{Geometrical Relations of Hexagonal Prisms}

Maximum dimension $D$ is equal to $2 a_{\text {hex }}$ for plates and $2 \alpha a_{\text {hex }}$ for columns, where $a_{\text {hex }}$ is the side length of the hexagon, which is the same as the maximum half-width of the hexagonal planes, and $\alpha$ is aspect ratio, determined throughout as the ratio of prism height to width.

Volume $V$ is related to $a_{\text {hex }}$ and $\alpha$ through

$$
V=3 \sqrt{3} a_{\text {hex }}^{3} \alpha .
$$

Since a hexagonal structure is a convex particle, the project area assuming random orientation $A_{p}$ is equal to the total surface area divided by 4 (Vouk 1948):

$$
A_{p}=\frac{3 \sqrt{3} a_{\mathrm{hex}}^{2}+12 a_{\mathrm{hex}}^{2} \alpha}{4} .
$$

Effective distance $d_{e}$, determined through Eq. (4) in the main text, is then given by

$$
d_{e}=a_{\mathrm{hex}} \frac{4 \sqrt{3} \alpha}{\sqrt{3}+4 \alpha} .
$$

\section{APPENDIX B}

\section{Two-Stream Radiative Transfer}

We apply the two-stream approximation for radiative transfer (Coakley and Chylek 1975; Stephens et al. 2001). In this method, the reflectance and transmittance of a cloud layer with optical thickness $\tau$ are given by (see also Wiscombe and Grams 1976)

$$
R\left(\mu_{0}\right)=\frac{(U+1)(U-1)\left(e^{\gamma \tau / \mu_{0}}-e^{-\gamma \tau / \mu_{0}}\right)}{(U+1)^{2} e^{\gamma \tau / \mu_{0}}-(U-1)^{2} e^{-\gamma \tau / \mu_{0}}}
$$

and

$$
T\left(\mu_{0}\right)=\frac{4 U}{(U+1)^{2} e^{\gamma \tau / \mu_{0}}-(U-1)^{2} e^{-\gamma \tau / \mu_{0}},}
$$

where $\mu_{0}$ is the cosine of the solar zenith angle and

$$
U=\sqrt{\frac{1-\omega+\omega(1-g)}{1-\omega}},
$$




$$
\gamma=\sqrt{(1-\omega)[1-\omega+\omega(1-g)]} .
$$

At nonabsorbing wavelengths $(\omega=1)$, this simplifies to

$$
R\left(\mu_{0}\right)=\frac{\tau(1-g)}{2 \mu_{0}+\tau(1-g)}
$$

and $T=1-R$.

The cloud layer can be embedded in a Rayleighscattering and absorbing atmosphere. Ignoring surface reflection and multiple scattering, the monochromatic upwelling flux $F_{\uparrow}$ at the top of atmosphere with a cloud layer whose top is located at optical depth $\tau_{t}$ is given by

$$
F_{\uparrow}=F_{\odot} e^{-\tau_{t} / \mu_{0}} \int_{0}^{1} \mathbf{R}\left(\mu, \mu_{0}\right) e^{-\tau_{t} / \mu} \mu d \mu,
$$

where $F_{\odot}$ is the monochromatic solar flux, $\mu$ is the angle of outgoing radiation, and $\mathbf{R}\left(\mu, \mu_{0}\right)$ is a bidirectional reflection matrix. To evaluate this integral, we use the approximations $\mathbf{R}\left(\mu, \mu_{0}\right)=R\left(\mu_{0}\right)$ and

$$
\int_{0}^{1} \mu e^{-\tau_{t} / \mu} d \mu \approx e^{-\tau_{t} / \mu_{\mathrm{eff}}},
$$

where $\mu_{\text {eff }}=1 / \sqrt{3}$, leading to

$$
F_{\uparrow}=F_{\odot} R\left(\mu_{0}\right) e^{-\tau_{t}\left(1 / \mu_{0}+1 / \mu_{\text {eff }}\right)} .
$$

Similarly, the downwelling diffuse flux $F_{\downarrow}$ at the surface is given by

$$
F_{\downarrow}=F_{\odot} T\left(\mu_{0}\right) e^{-\tau_{t} / \mu_{0}} e^{-\left(\tau_{\text {tot }}-\tau_{b}\right) / \mu_{\text {eff }}},
$$

where $\tau_{b}$ is the optical depth level at the bottom of the cloud layer and $\tau_{\text {tot }}$ is the total optical thickness of the atmosphere excluding the cloud layer. Note that extinction from gaseous absorption and Rayleigh scattering within the cloud layer is ignored by Eq. (B9). Cloud absorptance $a_{\text {cloud }}$ is defined as

$$
a_{\text {cloud }}=\left[F_{\odot}-R\left(\mu_{0}\right)-T\left(\mu_{0}\right)\right] e^{-\tau_{t} / \mu_{0}} .
$$

For wavelength bands that include high spectrally variant absorption lines, the correlated- $k$ distribution method (Lacis and Oinas 1991) is used.

\section{REFERENCES}

Auer, A., and D. Veal, 1970: The dimension of ice crystals in natural clouds. J. Atmos. Sci., 27, 919-926, doi:10.1175/ 1520-0469(1970)027<0919:TDOICI >2.0.CO;2.
Baker, B., and R. P. Lawson, 2006: Improvement in determination of ice water content from two-dimensional particle imagery. Part I: Image-to-mass relationships. J. Appl. Meteor. Climatol., 45, 1282-1290.

Baran, A. J., 2009: A review of the light scattering properties of cirrus. J. Quant. Spectrosc. Radiat. Transfer, 110 (14-16), 1239-1260, doi:10.1016/j.jqsrt.2009.02.026.

_ 2012: From the single-scattering properties of ice crystals to climate prediction: A way forward. Atmos. Res., 112, 45-69, doi:10.1016/j.atmosres.2012.04.010.

Baum, B. A., A. J. Heymsfield, P. Yang, and S. T. Bedka, 2005a: Bulk scattering properties for the remote sensing of ice clouds. Part I: Microphysical data and models. J. Appl. Meteor., 44, 1885-1895, doi:10.1175/JAM2308.1.

, P. Yang, A. J. Heymsfield, S. Platnick, M. D. King, Y. X. Hu, and S. M. Bedka, 2005b: Bulk scattering properties for the remote sensing of ice clouds. Part II: Narrowband models. J. Appl. Meteor., 44, 1896-1911, doi:10.1175/JAM2309.1.

Böhm, J. P., 1989: A general equation for the terminal fall speed of solid hydrometeors. J. Atmos. Sci., 46, 2419-2427.

— 1992: A general hydrodynamic theory for mixed-phase microphysics. Part I: Drag and fall speed of hydrometeors. Atmos. Res., 27, 253-274, doi:10.1016/0169-8095(92)90035-9.

Born, M., and E. Wolf, 1999: Principles of Optics. 7th ed. Cambridge University Press, 985 pp.

Bryant, F., and P. Latimer, 1969: Optical efficiencies of large particles of arbitrary shape and orientation. J. Colloid Interface Sci., 30, 291-304, doi:10.1016/0021-9797(69)90396-8.

Chepfer, H., P. Goloub, J. Riedi, J. De Haan, J. Hovenier, and P. Flamant, 2001: Ice crystal shapes in cirrus clouds derived from POLDER/ADEOS-1. J. Geophys. Res., 106 (D8), 7955 7966, doi:10.1029/2000JD900285.

Coakley, J. A. J., and P. Chylek, 1975: The two-stream approximation in radiative transfer: Including the angle of the incident radiation. J. Atmos. Sci., 32, 409-418, doi:10.1175/ 1520-0469(1975)032<0409:TTSAIR > 2.0.CO;2.

Edwards, J., S. Havemann, J.-C. Thelen, and A. Baran, 2007: A new parametrization for the radiative properties of ice crystals: Comparison with existing schemes and impact in a GCM. Atmos. Res., 83, 19-35, doi:10.1016/j.atmosres.2006.03.002.

Foot, J. S., 1988: Some observations of the optical properties of clouds. II: Cirrus. Quart. J. Roy. Meteor. Soc., 114, 145-164, doi:10.1002/qj.49711447908.

Francis, P. N., A. Jones, R. W. Saunders, K. P. Shine, A. Slingo, and Z. Sun, 1994: An observational and theoretical study of the radiative properties of cirrus: Some results from ICE'89. Quart. J. Roy. Meteor. Soc., 120, 809-848, doi:10.1002/ qj. 49712051804 .

Fridlind, A., and Coauthors, 2012: A comparison of TWP-ICE observational data with cloud-resolving model results. J. Geophys. Res., 117, D05204, doi:10.1029/2011JD016595.

$\mathrm{Fu}$, Q., 1996: An accurate parameterization of the solar radiative properties of cirrus clouds for climate models. J. Climate, 9, 2058-2082, doi:10.1175/1520-0442(1996)009<2058: AAPOTS $>2.0 . \mathrm{CO} ; 2$.

_ 2007: A new parameterization of an asymmetry factor of cirrus clouds for climate models. J. Atmos. Sci., 64, 4140-4150, doi:10.1175/2007JAS2289.1.

— , and K. N. Liou, 1993: Parameterization of the radiative properties of cirrus clouds. J. Atmos. Sci., 50, 2008-2025, doi:10.1175/1520-0469(1993)050<2008:POTRPO > 2.0.CO;2.

- W. B. Sun, and P. Yang, 1999: Modeling of scattering and absorption by nonspherical cirrus ice particles at thermal 
infrared wavelengths. J. Atmos. Sci., 56, 2937-2947, doi:10.1175/1520-0469(1999)056<2937:MOSAAB > 2.0.CO;2.

Garrett, T. J., P. V. Hobbs, and H. Gerber, 2001: Shortwave, singlescattering properties of arctic ice clouds. J. Geophys. Res., 106 (D14), 15 155-15172, doi:10.1029/2000JD900195.

Grenfell, T. C., and S. G. Warren, 1999: Representation of a nonspherical ice particle by a collection of independent spheres for scattering and absorption of radiation. J. Geophys. Res., 104 (D24), 31 697-31 709, doi:10.1029/1999JD900496.

Guimarães, L., and H. Nussenzveig, 1992: Theory of Mie resonances and ripple fluctuations. Opt. Commun., 89 (5-6), 363369, doi:10.1016/0030-4018(92)90540-8.

Hess, M., R. B. Koelemeijer, and P. Stammes, 1998: Scattering matrices of imperfect hexagonal ice crystals. J. Quant. Spectrosc. Radiat. Transfer, 60, 301-308, doi:10.1016/ S0022-4073(98)00007-7.

Heymsfield, A. J., and J. Iaquinta, 2000: Cirrus crystal terminal velocities. J. Atmos. Sci., 57, 916-938, doi:10.1175/ 1520-0469(2000)057<0916:CCTV>2.0.CO;2.

—, A. Bansemer, P. R. Field, S. L. Durden, J. L. Stith, J. E. Dye, W. Hall, and C. A. Grainger, 2002: Observations and parameterizations of particle size distributions in deep tropical cirrus and stratiform precipitating clouds: Results from in situ observations in TRMM field campaigns. J. Atmos. Sci., 59, 3457-3491, doi:10.1175/1520-0469(2002)059<3457: OAPOPS $>2.0 . \mathrm{CO} ; 2$.

Iaquinta, J., H. Isaka, and P. Personne, 1995: Scattering phase function of bullet rosette ice crystals. J. Atmos. Sci., 52, 1401-1413, doi:10.1175/1520-0469(1995)052<1401:SPFOBR > 2.0.CO;2.

Kato, S., T. P. Ackerman, J. H. Mather, and E. E. Clothiaux, 1999: The k-distribution method and correlated-k approximation for a shortwave radiative transfer model. J. Quant. Spectrosc. Radiat. Transfer, 62, 109-121, doi:10.1016/S0022-4073(98)00075-2.

Key, J. R., P. Yang, B. A. Baum, and S. Nasiri, 2002: Parameterization of shortwave ice cloud optical properties for various particle habits. J. Geophys. Res., 107, 4181, doi:10.1029/ 2001JD000742.

King, M. D., S. Platnick, P. Yang, G. T. Arnold, M. A. Gray, J. C. Riedi, S. A. Ackerman, and K.-N. Liou, 2004: Remote sensing of liquid water and ice cloud optical thickness and effective radius in the Arctic: Application of airborne multispectral MAS data. J. Atmos. Oceanic Technol., 21, 857-875, doi:10.1175/1520-0426(2004)021<0857:RSOLWA >2.0.CO;2.

Korolev, A. V., and G. Isaac, 2003: Roundness and aspect ratio of particles in ice clouds. J. Atmos. Sci., 60, 1795-1808, doi:10.1175/ 1520-0469(2003)060<1795:RAAROP $>2.0$. CO;2.

—, E. F. Emery, J. W. Strapp, S. G. Cober, G. A. Isaac, M. Wasey, and D. Marcotte, 2011: Small ice particles in tropospheric clouds: fact or artifact? Airborne icing instrumentation evaluation experiment. Bull. Amer. Meteor. Soc., 92, 967-973, doi:10.1175/ 2010BAMS3141.1.

Kristjánsson, J. E., J. Edwards, and D. Mitchell, 1999: A new parameterization scheme for the optical properties of ice crystals for use in general circulation models of the atmosphere. Phys. Chem. Earth, 24B, 231-236, doi:10.1016/ S1464-1909(98)00043-4.

,-- , and - , 2000: Impact of a new scheme for optical properties of ice crystals on climates of two GCMs. J. Geophys. Res., 105 (D8), 10063-10 079, doi:10.1029/2000JD900015.

Lacis, A. A., and V. Oinas, 1991: A description of the correlated $k$ distribution method for modeling nongray gaseous absorption, thermal emission, and multiple scattering in vertically inhomogeneous atmospheres. J. Geophys. Res., 96 (D5), 90279063, doi:10.1029/90JD01945.

Lawson, R. P., E. Jensen, D. L. Mitchell, B. Baker, Q. Mo, and B. Pilson, 2010: Microphysical and radiative properties of tropical clouds investigated in TC4 and NAMMA. J. Geophys. Res., 115, D00J08, doi:10.1029/2009JD013017.

Lindqvist, H., K. Muinonen, T. Nousiainen, J. Um, G. M. McFarquhar, P. Haapanala, R. Makkonen, and H. Hakkarainen, 2012: Icecloud particle habit classification using principal components. J. Geophys. Res., 117, D16206, doi:10.1029/2012JD017573.

Liu, X., S. Ding, L. Bi, and P. Yang, 2012: On the use of scattering kernels to calculate ice cloud bulk optical properties. J. Atmos. Oceanic Technol., 29, 50-63, doi:10.1175/JTECH-D-11-00034.1.

Macke, A., M. I. Mishchenko, K. Muinonen, and B. E. Carlson, 1995: Scattering of light by large nonspherical particles: Raytracing approximation versus T-matrix method. Opt. Lett., 20, 1934-1936, doi:10.1364/OL.20.001934.

_, J. Mueller, and E. Raschke, 1996: Single scattering properties of atmospheric ice crystals. J. Atmos. Sci., 53, 2813-2825, doi:10.1175/1520-0469(1996)053<2813:SSPOAI >2.0.CO;2.

Mauno, P., G. M. McFarquhar, P. Räisänen, M. Kahnert, M. S. Timlin, and T. Nousiainen, 2011: The influence of observed cirrus microphysical properties on shortwave radiation: A case study over Oklahoma. J. Geophys. Res., 116, D22208, doi:10.1029/2011JD016058.

McFarquhar, G. M., and A. J. Heymsfield, 1998: The definition and significance of an effective radius for ice clouds. J. Atmos. Sci., 55, 2039-2052, doi:10.1175/1520-0469(1998)055<2039: TDASOA $>2.0 . \mathrm{CO} ; 2$.

_- P. Yang, A. Macke, and A. J. Baran, 2002: A new parameterization of single scattering solar radiative properties for tropical anvils using observed ice crystal size and shape distributions. J. Atmos. Sci., 59, 2458-2478, doi:10.1175/ 1520-0469(2002)059<2458:ANPOSS > 2.0.CO;2.

Mishchenko, M. I., and A. Macke, 1998: Incorporation of physical optics effects and computation of the Legendre expansion for ray-tracing phase functions involving $\delta$-function transmission. J. Geophys. Res., 103 (D2), 1799-1805, doi:10.1029/ 97JD03121.

Mitchell, D. L., 1991: Evolution of snow-size spectra in cyclonic storms. Part II: Deviations from the exponential form. J. Atmos. Sci., 48, 1885-1899, doi:10.1175/1520-0469(1991)048<1885: EOSSSI $>2.0 . \mathrm{CO} ; 2$.

—, 2000: Parameterization of the Mie extinction and absorption coefficients for water clouds. J. Atmos. Sci., 57, 1311-1326, doi:10.1175/1520-0469(2000)057<1311:POTMEA > 2.0.CO;2.

_ 2002: Effective diameter in radiation transfer: General definition, applications, and limitations. J. Atmos. Sci., 59, 2330-2346, doi:10.1175/1520-0469(2002)059<2330:EDIRTG >2.0.CO;2.

- and W. P. Arnott, 1994: A model predicting the evolution of ice particle size spectra and radiative properties of cirrus clouds. Part II: Dependence of absorption and extinction on ice crystal morphology. J. Atmos. Sci., 51, 817-832, doi:10.1175/ 1520-0469(1994)051<0817:AMPTEO > 2.0.CO;2.

, Y. Liu, and A. Macke, 1996: Modeling cirrus clouds. Part II: Treatment of radiative properties. J. Atmos. Sci., 53, 2967-2988, doi:10.1175/1520-0469(1996)053<2967:MCCPIT>2.0.CO;2.

_ A. J. Baran, W. P. Arnott, and C. Schmitt, 2006: Testing and comparing the modified anomalous diffraction approximation. J. Atmos. Sci., 63, 2948-2962, doi:10.1175/ JAS3775.1.

-, P. Rasch, D. Ivanova, G. McFarquhar, and T. Nousiainen, 2008: Impact of small ice crystal assumptions on ice 
sedimentation rates in cirrus clouds and GCM simulations. Geophys. Res. Lett., 35, L09806, doi:10.1029/2008GL033552.

Neshyba, S. P., T. C. Grenfell, and S. G. Warren, 2003: Representation of a nonspherical ice particle by a collection of independent spheres for scattering and absorption of radiation: 2. Hexagonal columns and plates. J. Geophys. Res., 108, 4448, doi:10.1029/2002JD003302.

- B. Lowen, M. Benning, A. Lawson, and P. M. Rowe, 2013: Roughness metrics of prismatic facets of ice. J. Geophys. Res., 118, 3309-3318, doi:10.1002/jgrd.50357.

Schlimme, I., A. Macke, and J. Reichardt, 2005: The impact of ice crystal shapes, size distributions, and spatial structures of cirrus clouds on solar radiative fluxes. J. Atmos. Sci., 62, 2274 2283, doi:10.1175/JAS3459.1.

Stackhouse, P. W. J., and G. L. Stephens, 1991: A theoretical and observational study of the radiative properties of cirrus: Results from FIRE 1986. J. Atmos. Sci., 48, 2044-2059, doi:10.1175/1520-0469(1991)048<2044:ATAOSO > 2.0.CO;2.

Stephens, G. L., S.-C. Tsay, J. Stackhouse, W. Paul, and P. J. Flatau, 1990: The relevance of the microphysical and radiative properties of cirrus clouds to climate and climatic feedback. $J$. Atmos. Sci., 47, 1742-1754, doi:10.1175/1520-0469(1990)047<1742: TROTMA $>2.0 . \mathrm{CO} ; 2$.

— , P. M. Gabriel, and P. T. Partain, 2001: Parameterization of atmospheric radiative transfer. Part I: Validity of simple models. J. Atmos. Sci., 58, 3391-3409, doi:10.1175/ 1520-0469(2001)058<3391:POARTP >2.0.CO;2.

Sulia, K. J., and J. Y. Harrington, 2011: Ice aspect ratio influences on mixed-phase clouds: Impacts on phase partitioning in parcel models. J. Geophys. Res., 116, D21309, doi:10.1029/ 2011JD016298.

Takano, Y., and K.-N. Liou, 1989: Solar radiative transfer in cirrus clouds. Part I: Single-scattering and optical properties of hexagonal ice crystals. J. Atmos. Sci., 46, 3-19, doi:10.1175/ 1520-0469(1989)046<0003:SRTICC $>2.0$. CO;2.

Um, J., and G. M. McFarquhar, 2007: Single-scattering properties of aggregates of bullet rosettes in cirrus. J. Appl. Meteor. Climatol., 46, 757-775, doi:10.1175/JAM2501.1.

$\longrightarrow$, and -2009 : Single-scattering properties of aggregates of plates. Quart. J. Roy. Meteor. Soc., 135, 291-304, doi:10.1002/ qj.378.

van de Hulst, H. C., 1957: Light Scattering by Small Particles. Dover Publications, $480 \mathrm{pp}$

van Diedenhoven, B., B. Cairns, I. V. Geogdzhayev, A. M. Fridlind, A. S. Ackerman, P. Yang, and B. A. Baum, 2012a: Remote sensing of ice crystal asymmetry parameter using multidirectional polarization measurements-Part 1: Methodology and evaluation with simulated measurements. Atmos. Meas. Tech., 5, 2361-2374, doi:10.5194/amt-5-2361-2012.

, A. M. Fridlind, A. S. Ackerman, and B. Cairns, 2012b: Evaluation of hydrometeor phase and ice properties in cloudresolving model simulations of tropical deep convection using radiance and polarization measurements. J. Atmos. Sci., 69, 3290-3314, doi:10.1175/JAS-D-11-0314.1.

, B. Cairns, A. M. Fridlind, A. S. Ackerman, and T. J. Garrett, 2013: Remote sensing of ice crystal asymmetry parameter using multi-directional polarization measurements-Part 2: Application to the Research Scanning Polarimeter. Atmos. Chem. Phys., 13, 3185-3203, doi:10.5194/acp-13-3185-2013.
Vouk, V., 1948: Projected area of convex bodies. Nature, 162, 330 331, doi:10.1038/162330a0.

Warren, S. G., 1984: Optical constants of ice from the ultraviolet to the microwave. Appl. Opt., 23, 1206-1225, doi:10.1364/ AO.23.001206.

- , and R. E. Brandt, 2008: Optical constants of ice from the ultraviolet to the microwave: A revised compilation. J. Geophys. Res., 113, D14220, doi:10.1029/2007JD009744.

Westbrook, C. D., 2008: The fall speeds of sub-100 micron ice crystals. Quart. J. Roy. Meteor. Soc., 134, 1243-1251, doi:10.1002/ qj.290.

_ R. J. Hogan, and A. J. Illingworth, 2008: The capacitance of pristine ice crystals and aggregate snowflakes. J. Atmos. Sci., 65, 206-219, doi:10.1175/2007JAS2315.1.

Wiscombe, W., and G. Grams, 1976: The backscattered fraction in two-stream approximations. J. Atmos. Sci., 33, 2440-2451, doi:10.1175/1520-0469(1976)033<2440:TBFITS > 2.0.CO;2.

Wood, S. E., M. B. Baker, and D. Calhoun, 2001: New model for the vapor growth of hexagonal ice crystals in the atmosphere. J. Geophys. Res., 106, 4845-4870, doi:10.1029/2000JD900338.

Wyser, K., and P. Yang, 1998: Average ice crystal size and bulk short-wave single-scattering properties of cirrus clouds. Atmos. Res., 49, 315-335, doi:10.1016/S0169-8095(98)00083-0.

Xie, Y., P. Yang, K. Liou, P. Minnis, and D. Duda, 2012: Parameterization of contrail radiative properties for climate studies. Geophys. Res. Lett., 39, L00F02, doi:10.1029/2012GL054043.

Yang, P., and K. N. Liou, 1996: Geometric-optics integral-equation method for light scattering by nonspherical ice crystals. Appl. Opt., 35, 6568-6584, doi:10.1364/AO.35.006568.

— crystals in terrestrial atmosphere. Contrib. Atmos. Phys., 71, 223-248.

—, and Q. Fu, 2009: Dependence of ice crystal optical properties on particle aspect ratio. J. Quant. Spectrosc. Radiat. Transfer, 110 (14-16), 1604-1614, doi:10.1016/j.jqsrt.2009.03.004.

— , K. Liou, K. Wyser, and D. Mitchell, 2000: Parameterization of scattering and absorption properties of individual ice crystals. J. Geophys. Res., 105 (D4), 4699-4718, doi:10.1029/ 1999JD900755.

— , Z. Zhang, B. A. Baum, H.-L. Huang, and Y. Hu, 2004: A new look at anomalous diffraction theory (ADT): Algorithm in cumulative projected-area distribution domain and modified ADT. J. Quant. Spectrosc. Radiat. Transfer, 89 (1-4), 421-442, doi:10.1016/j.jqsrt.2004.05.038.

—, G. Kattawar, G. Hong, P. Minnis, and Y. Hu, 2008: Uncertainties associated with the surface texture of ice particles in satellite-based retrieval of cirrus clouds-Part I: Singlescattering properties of ice crystals with surface roughness. IEEE Trans. Geosci. Remote Sens., 46, 1940-1947, doi:10.1109/ TGRS.2008.916471.

—, L. Bi, B. A. Baum, K.-N. Liou, G. W. Kattawar, M. I. Mishchenko, and B. Cole, 2013: Spectrally consistent scattering, absorption, and polarization properties of atmospheric ice crystals at wavelengths from $0.2 \mu \mathrm{m}$ to $100 \mu \mathrm{m}$. J. Atmos. Sci., 70, 330-347, doi:10.1175/JAS-D-12-039.1.

Yi, B., P. Yang, B. A. Baum, T. L'Ecuyer, L. Oreopoulos, E. J. Mlawer, A. J. Heymsfield, and K.-N. Liou, 2013: Influence of ice particle surface roughening on the global cloud radiative effect. J. Atmos. Sci., 70, 2794-2807, doi:10.1175/JAS-D-13-020.1. 